\title{
Self-dual 6d 2-form fields coupled to non-abelian gauge field: quantum corrections
}

\author{
Kuo-Wei Huang, ${ }^{a, b}$ Radu Roiban ${ }^{c}$ and Arkady A. Tseytlin ${ }^{d, 1}$ \\ ${ }^{a}$ C.N. Yang Institute for Theoretical Physics, \\ Stony Brook University, Stony Brook, NY 11794, U.S.A. \\ ${ }^{b}$ Perimeter Institute for Theoretical Physics, \\ Waterloo, Ontario N2L 2Y5, Canada \\ ${ }^{c}$ Department of Physics, The Pennsylvania State University, \\ University Park, PA 16802, U.S.A. \\ ${ }^{d}$ Blackett Laboratory, Imperial College, \\ London $S W^{\text {ry }} 2 A Z$, U.K. \\ E-mail: kuo-wei.huang@stonybrook.edu, radu@phys.psu.edu, \\ tseytlin@imperial.ac.uk
}

ABSTRACT: We study a $6 \mathrm{~d}$ model of a set of self-dual 2-form $B$-fields interacting with a non-abelian vector $A$-field which is restricted to a $5 \mathrm{~d}$ subspace. One motivation is that if the gauge vector could be expressed in terms of the $B$-field or integrated out, this model could lead to an interacting theory of $B$-fields only. Treating the 5 d gauge vector as a background field, we compute the divergent part of the corresponding one-loop effective action which has the $(D F)^{2}+F^{3}$ structure and compare it with similar contributions from other $6 \mathrm{~d}$ fields. We also discuss a $4 \mathrm{~d}$ analog of the non-abelian self-dual model, which turns out to be UV finite.

Keywords: Anomalies in Field and String Theories, Field Theories in Higher Dimensions, Gauge Symmetry

ARXIV EPRINT: 1804.05059

\footnotetext{
${ }^{1}$ Also at Lebedev Institute, Moscow.
} 


\section{Contents}

1 Introduction 1

2 Non-abelian $B$-field coupled to gauge vector $\quad 3$

2.1 Gauge symmetry and field strength 3

2.2 Classical action and gauge fixing 5

2.3 Self-dual $B$-field model 6

3 Structure of divergent part of effective action 9

4 Calculation of one-loop divergences $\quad 10$

$\begin{array}{lll}4.1 & \text { Self-dual } B \text {-field model } & 10\end{array}$

$\begin{array}{lll}4.1 .1 & A^{2} \text { term } & 11\end{array}$

$\begin{array}{lll}4.1 .2 & A^{3} \text { term } & 12\end{array}$

$\begin{array}{lll}4.2 & \text { Non-chiral } B \text {-field model } & 14\end{array}$

$\begin{array}{lll}4.2 .1 & A^{2} \text { term } & 14\end{array}$

$\begin{array}{lll}4.2 .2 & A^{3} \text { term } & 15\end{array}$

5 Concluding remarks $\quad 16$

$\begin{array}{ll}\text { A Free partition function } & 17\end{array}$

$\begin{array}{lr}\text { B Useful integrals } & 18\end{array}$

$\begin{array}{lll}\text { C } & A^{6} \text { term in effective action of self-dual B-field } & 19\end{array}$

$\begin{array}{ll}\text { D Non-abelian chiral 4d vector model } & 20\end{array}$

\section{Introduction}

The possible existence of interacting theories of non-abelian 2-form fields in 6 dimensions possessing some unusual properties such as lack of manifest Lorentz symmetry and/or locality is an important open problem (for a recent review and references see, e.g., [1]). As a first step, one may study a system of 2 -form potentials $B$ in some representation of gauge group $G$ coupled "minimally" to a non-abelian gauge vector $A$. Similar couplings appeared, e.g., in the context of attempts to construct an interacting theory of $6 \mathrm{~d}(2,0)$ tensor multiplets in [2-4] (see also [5-7]).

Here we shall consider a simple bosonic model of interacting $(B, A)$ fields following [8-11]. We shall study both the model with self-dual $B$-field strength and the non-chiral $B$-field model. It turns out that a consistent gauge-invariant coupling is possible provided 
one keeps only the $5 \mathrm{~d}$ part of the $6 \mathrm{~d}$ Lorentz symmetry. ${ }^{1}$ The action is quadratic in $B$ and takes a local form in a particular gauge, with the $A$-field restricted to "live" only in $5 \mathrm{~d}$ subspace of the $6 \mathrm{~d}$ space. More generally, one may attempt to consider an extension where $A$ is expressed in terms of $B$ leading to a non-local interacting theory of $B$-fields only.

Our aim will be to study this $(B, A)$ model at the quantum level. ${ }^{2}$ We shall concentrate on the one-loop approximation where $B$ is integrated out and $A$ is treated as a background. As is well known, quantizing free scalar, spinor or Yang-Mills (YM) fields coupled to an external vector in 6 dimensions produces $(D F)^{2}+F^{3}$ logarithmic UV divergences in the effective action (see, e.g., [12]). We shall find that similar divergences appear also from the $B$-field loop, implying, in particular, the breaking of the classical scale invariance. One may hope to cancel these divergences by adding other fields (e.g., imposing supersymmetry) but so far we did not find such a finite model.

As in the case of the $6 \mathrm{~d}$ Weyl fermions [13, 14], one could expect that the chiral nature of the self-dual $B$-field model implies the presence of anomalous (gauge-symmetry breaking) terms in the parity-odd part of the effective action (which would be a gaugefield counterpart of the familiar gravitational anomaly in the case of a single self-dual tensor $[14,15])$. However, this does not happen in the present case: as the $A$-field is restricted to 5 dimensions, the effective action has no parity-odd part, i.e. there is no gauge anomaly as in any 5 d theory.

We start in section 2 with a description of the gauge symmetries and the classical action of the $(B, A)$ model - both its non-chiral version and the chiral version with the self-dual $B$-field strength. In the $B_{i 6}=0$ gauge the corresponding actions take simple form (2.14) and (2.27). We shall argue that the one-loop effective action of non-chiral model (2.31) should be twice the effective action of the self-dual model. ${ }^{3}$

The general $(D F)^{2}+F^{3}$ structure (3.2) of the UV divergent part of the 6d effective action in a gauge field background will be discussed in section 3 . We shall summarize the results for the corresponding two coefficients $\beta_{2}$ and $\beta_{3}$ for a collection of $6 \mathrm{~d}$ fields (see (3.6), (3.7)).

The values of $\beta_{2}$ and $\beta_{3}$ for the self-dual and the non-chiral models will be derived in detail in section 4 by computing the divergent parts of the $A^{2}$ and $A^{3}$ terms in the effective action. We shall use dimensional regularization procedure (applied only with respect to 5 -momenta) that preserves background gauge invariance.

Some concluding remarks will be made in section 5. In appendix A we review the structure of the free $B$-field partition function. Some standard integrals are summarized in appendix B. The same values of $\beta_{2}$ (4.12) and $\beta_{3}$ (4.19) in the self-dual model are independently obtained in appendix $\mathrm{C}$ from the $A^{6}$ term in the effective action. In appendix $\mathrm{D}$ we discuss a non-local effective action of a $4 \mathrm{~d}$ analog of the $6 \mathrm{~d}$ self-dual $(B, A)$ model.

\footnotetext{
${ }^{1}$ This may not be unnatural given that already at the free level the Lagrangian description of a self-dual $B$-field is not manifestly Lorentz invariant.

${ }^{2}$ The model of [8] in the generalized version adopted below has an advantage of having an explicit Lagrangian formulation for massless $6 \mathrm{~d} 2$-forms without introducing extra auxiliary fields. It would be interesting also to perform a quantum study of similar models considered in refs. [1-7].

${ }^{3}$ If the effective action of the chiral model had a parity-odd component, the effective action of the non-chiral model would be twice the parity-even part of the chiral effective action.
} 


\section{Non-abelian $B$-field coupled to gauge vector}

\subsection{Gauge symmetry and field strength}

The abelian antisymmetric rank 2 tensor field has a familiar gauge symmetry

$$
\delta B_{\mu \nu}=\partial_{\mu} \epsilon_{\nu}-\partial_{\nu} \epsilon_{\mu}
$$

There is the residual gauge symmetry, $\delta \epsilon_{\mu}=\partial_{\mu} \eta$, which allows one to remove one component from $\epsilon_{\mu}$ and is thus important for the correct degrees of freedom count. A non-abelian generalization of (2.1) should also admit some non-abelian analog of this residual gauge symmetry. The abelian gauge-invariant 3-form field strength is $H_{\mu \nu \lambda}=\partial_{\mu} B_{\nu \lambda}+\partial_{\nu} B_{\lambda \mu}+$ $\partial_{\lambda} B_{\mu \nu}$. To write down a gauge-invariant action in a non-abelian case, there should exist a generalized field strength that transforms covariantly.

It turns out that it is possible to construct such a model if one relaxes the condition of $6 \mathrm{~d}$ Lorentz covariance (and locality). Our starting point will be a model involving a $6 \mathrm{~d}$ 2 -form field $B_{\mu \nu}$ in some representation of gauge group $G$ and a gauge vector field $A_{\mu}$. For the simplicity, we assume that both $B_{\mu \nu}$ and $A_{\mu}$ are taken in the adjoint representation of $G$ and use the following notation: $D_{\mu} \ldots=\partial_{\mu} \ldots+\left[A_{\mu}, \ldots\right], F_{\mu \nu}=\partial_{\mu} A_{\nu}-\partial_{\nu} A_{\mu}+\left[A_{\mu}, A_{\nu}\right]$. One can define the non-abelian gauge transformations as $[8]$

$$
\begin{aligned}
\delta A_{\mu} & =D_{\mu} \lambda \\
\delta B_{\mu \nu} & =D_{\mu} \epsilon_{\nu}-D_{\nu} \epsilon_{\mu}-\left[F_{\mu \nu},\left(n^{\rho} \partial_{\rho}\right)^{-1}\left(n^{\sigma} \epsilon_{\sigma}\right)\right]+\left[B_{\mu \nu}, \lambda\right] .
\end{aligned}
$$

Here $\lambda$ is the parameter of $A_{\mu}$ gauge transformations under which $B_{\mu \nu}$ transforms covariantly; $\epsilon_{\mu}$ is the parameter of the gauge transformations of $B_{\mu \nu}$, which, like $\lambda$, is now taking values in the algebra of $G$. The vector $n_{\mu}$ is a fixed constant unit vector which selects a particular direction in $6 \mathrm{~d}$ space breaking $O(6)$ symmetry to $O(5)$. In the abelian limit, the gauge transformation (2.3) reduces to $(2.1)$.

The structure of the non-local term in (2.3) is chosen to be such that if we further assume that $n^{\mu} A_{\mu}=0$ then there is a non-abelian generalization of the residual gauge symmetry of the parameter $\epsilon_{\mu}$ in (2.1) under which $\delta B_{\mu \nu}$ is invariant:

$$
\delta \epsilon_{\mu}=D_{\mu} \eta, \quad \delta \lambda=0 .
$$

If we impose the additional condition that $n^{\mu} \partial_{\mu} A_{\nu}=0$, i.e. that $A_{\mu}$ depends only on 5 of the 6 coordinates (so that, in particular, $\left[\left(n^{\mu} \partial_{\mu}\right)^{-1}, D_{\nu}\right] f=0$ for a 6 d function $\left.f\left(x_{\lambda}\right)\right)$ then one can check that the gauge algebra closes:

$$
\left[\delta_{1}, \delta_{2}\right]=\delta_{3}, \quad \text { with } \quad \lambda_{3}=\left[\lambda_{1}, \lambda_{2}\right], \quad \epsilon_{\mu 3}=\left[\lambda_{1}, \epsilon_{\mu 2}\right]-\left[\lambda_{2}, \epsilon_{\mu 1}\right]
$$

The corresponding field strength of $B_{\mu \nu}$ is defined as

$$
\begin{aligned}
H_{\mu \nu \lambda}= & D_{\mu} B_{\nu \lambda}+D_{\nu} B_{\lambda \mu}+D_{\lambda} B_{\mu \nu} \\
& +\left[F_{\mu \nu},\left(n^{\rho} \partial_{\rho}\right)^{-1}\left(n^{\sigma} B_{\lambda \sigma}\right)\right]+\left[F_{\nu \lambda},\left(n^{\rho} \partial_{\rho}\right)^{-1}\left(n^{\sigma} B_{\mu \sigma}\right)\right]+\left[F_{\lambda \mu},\left(n^{\rho} \partial_{\rho}\right)^{-1}\left(n^{\sigma} B_{\nu \sigma}\right)\right],
\end{aligned}
$$




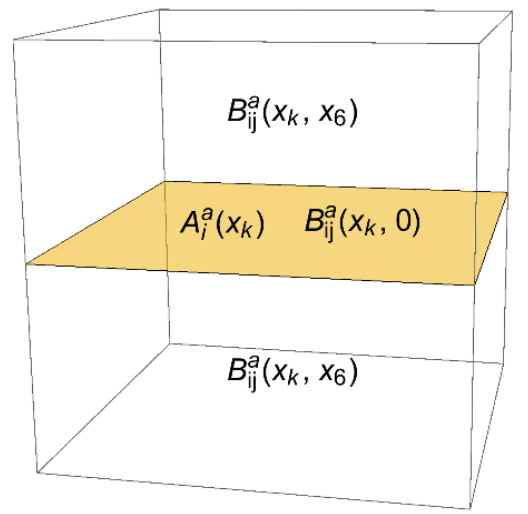

Figure 1. Sketch of $B$ - and $A$-fields in $6 \mathrm{~d}$ space. The $B$-field has 5 -indices (in $B_{i 6}=0$ gauge) but depends on all 6 coordinates. The $A$-field "lives" only in a codimension- 1 subspace with $x^{6}=0$ (colored region) where the interaction takes place.

where the non-local terms ensure that $H$ transforms covariantly:

$$
\delta H_{\mu \nu \sigma}=\left[H_{\mu \nu \sigma}, \lambda\right] .
$$

Thus one can consistently couple the non-abelian antisymmetric tensor to a non-abelian gauge field restricted to a codimension-1 ("boundary") subspace, i.e. with an effective nonlocality along the "bulk" direction (see figure 1). This non-locality may be viewed as a gauge artifact as there is a gauge in which the corresponding action is local (see below). Note also that we do not impose any boundary condition at $x_{6}=0$.

Without loss of generality, one can always choose $n_{\mu}$ to point in the 6 th direction, i.e. $n_{\mu}=(0,0,0,0,0,1)$, so that the vector field restricted as above "lives" in $5 \mathrm{~d}$ subspace ${ }^{4}$

$$
A_{\mu}=\left\{A_{i}\left(x^{k}, 0\right), A_{6}=0\right\}, \quad F_{i 6}=0, \quad F_{i j}=\partial_{i} A_{j}-\partial_{j} A_{i}+\left[A_{i}, A_{j}\right], \quad D_{6}=\partial_{6} .
$$

This $(B, A)$ model may be viewed as an intermediate step towards constructing an interacting model of $B$-fields only. For example, one may interpret $A$ not as an independent field but as related to $B$ by some non-local condition. In [8] the $x^{6}$ direction was assumed to be compactified to a circle of radius $R$ and $A_{i}$ was related to the zero mode of $B_{i 6}: A_{i} \equiv \int d x^{6} B_{i 6}=2 \pi R B_{i 6}^{\text {(zero mode) }}\left(x^{k}\right)$. Moreover, the zero mode of the 3 -form field strength was defined directly via the Hodge duality: $H_{i j k}\left(x^{k}, 0\right) \equiv \frac{i}{4 \pi R} \epsilon_{i j k m n} F_{m n} .{ }^{5}$ One may also treat $A_{i}$ first as an independent quantum field and then integrate it out in the path integral obtaining an effective non-local model of self-coupled non-abelian $B$-fields.

Below we shall view $A_{i}$ just as a background field coupled to the quantum $B$-fields. This interacting theory will have only $\mathrm{SO}(5)$ part of the full 6d rotational (Lorentz) symmetry.

\footnotetext{
${ }^{4}$ We assume the Euclidean signature with $6 \mathrm{~d}$ indices $\mu, \nu, \lambda, \ldots=1, \ldots, 6$ and use $i, j, k, \ldots=1, \ldots, 5$ for $5 \mathrm{~d}$ indices.

${ }^{5}$ Note that this implies $\int d^{6} x H_{i j k}^{2}\left(x^{k}, 0\right) \rightarrow \frac{1}{R} \int d^{5} x F_{i j}^{2}$, which is formally consistent with scaling symmetry. The compactification assumption naturally breaks the global $\mathrm{SO}(6)$ symmetry to $\mathrm{SO}(5) \times \mathrm{SO}(2)$. In the non-compact $x^{6}$ case one may set $A_{i} \equiv \int d x^{6} B_{i 6}, \quad \lambda \equiv \int d x^{6} \epsilon_{6}$ and impose the boundary conditions: $\eta\left(x_{i}, \pm \infty\right)=0, \epsilon_{i}\left(x_{k}, \pm \infty\right)=\left[A_{i}, \lambda\right]$, to preserve the gauge-covariant structure.
} 


\subsection{Classical action and gauge fixing}

Our starting point will be the following gauge invariant action describing the non-abelian 6 d field $B_{\mu \nu}\left(x^{\mu}\right)$ coupled to the 5 d gauge field $A_{i}\left(x^{j}\right)$ :

$$
S=\frac{1}{6} \int d^{6} x \operatorname{Tr}\left(H_{\mu \nu \lambda} H^{\mu \nu \lambda}\right) .
$$

Here $\operatorname{Tr}$ is in some representation $R$ of gauge group, with $\operatorname{Tr}\left(H^{a} t^{a}\right)^{2}=T_{R} H^{a} H^{a}(a=$ $1, \ldots, \operatorname{dim} G)$, with $t^{a}$ being hermitian generators and $T_{R}=\frac{1}{2}$ or $T_{R}=C_{2}$ if $H$ is a matrix with indices in fundamental or adjoint representation.

The overall (dimensionless) normalization constant in the action (2.9) will not be important as in this paper we will only consider the 1-loop approximation treating $A_{i}$ as a background field. In general, to make the model renormalizable one would need to introduce also $A$-dependent counterterms $\int d^{6} x\left[c_{1}(D F)^{2}+c_{2} F^{3}\right]$ (see below), i.e. two extra dimensionless coupling constants. ${ }^{6}$

From (2.6) and (2.8) we have

$$
H_{i j 6}=\partial_{6} B_{i j}+D_{i} B_{j 6}-D_{j} B_{i 6}, \quad H_{i j k}=D_{i} B_{j k}+\left[F_{i j}, \partial_{6}^{-1} B_{k 6}\right]+(i, j, k \text { cycle }) .
$$

The action (2.9) is invariant under gauge transformations (2.2) and (2.3), i.e.

$$
\delta B_{i j}=D_{i} \bar{\epsilon}_{j}-D_{j} \bar{\epsilon}_{i}+\left[B_{i j}, \lambda\right], \quad \delta B_{i 6}=-\partial_{6} \bar{\epsilon}_{i}+\left[B_{i 6}, \lambda\right], \quad \delta A_{i}=D_{i} \lambda
$$

where $\lambda=\lambda\left(x_{i}\right)$ and we redefined the gauge parameter $\epsilon_{i} \rightarrow \bar{\epsilon}_{i}$ as

$$
\bar{\epsilon}_{i}=\epsilon_{i}-D_{i} \partial_{6}^{-1} \epsilon_{6}
$$

We can fix the $\bar{\epsilon}_{i}$ gauge freedom by the natural gauge $B_{i 6}=0$ in which the field strength becomes manifestly local

$$
B_{i 6}=0: \quad H_{i j k}=D_{i} B_{j k}+D_{j} B_{k i}+D_{k} B_{i j}, \quad H_{i j 6}=\partial_{6} B_{i j}
$$

The gauge-fixed action (2.9) is then given by

$$
\begin{aligned}
S & =\frac{1}{2} \int d^{6} x \operatorname{Tr}\left[\left(\partial_{6} B_{i j}\right)^{2}+\frac{1}{3} H^{i j k} H_{i j k}\right]=\frac{1}{2} \int d^{6} x \operatorname{Tr}\left(B^{i j} \Delta_{i j}^{m n} B_{m n}\right), \\
\Delta_{i j}^{m n} & =-\delta_{i j}^{m n}\left(\partial_{6}^{2}+D^{2}\right)+2 \delta_{[i}^{[m} D^{n]} D_{j]}, \quad D^{2} \equiv D^{i} D_{i}, \quad \delta_{i j}^{m n}=\delta_{[i}^{m} \delta_{j]}^{n},
\end{aligned}
$$

where $[i j]$ stands for antisymmetrisation with weight $\frac{1}{2}$ and $D_{i} B_{j k} \equiv \partial_{i} B_{j k}+\left[A_{i}, B_{j k}\right]^{7}$

\footnotetext{
${ }^{6}$ In general, one could also consider adding the 5d Chern-Simons action for $A: S_{5 \mathrm{~d}}=\frac{\kappa}{3} \int \operatorname{Tr}(A \wedge F \wedge$ $F+\frac{i}{2} A \wedge A \wedge A \wedge F-\frac{1}{10} A \wedge A \wedge A \wedge A \wedge A$ ), but it will not be naturally induced in the model based on (2.9) and also in its self-dual version discussed below.

${ }^{7}$ Note that the $6 \mathrm{~d}$ action $(2.9)$ or $(2.14)$ is manifestly scale invariant. Starting with such an $(d B+A B)^{2}$ action and integrating out $A_{i}$ should give a local, scale-invariant but non-polynomial and non-Lorentzinvariant action $\sim(B B)^{-1} d B d B$ for the non-abelian $B$-fields. It will not have free quadratic part and will thus require some non-trivial (scale-invariance breaking) $B$-field background to define a perturbation theory (cf. [16]).
} 
Our aim will be to compute the logarithmic divergences in the $A_{i}$-dependent 1-loop effective action found by integrating out the $B$-field in $(2.14)^{8}$

$$
\Gamma=\frac{1}{2} \log \operatorname{det} \Delta_{i j}^{m n}(A) .
$$

The operator $\Delta_{i j}^{m n}$ in (2.15) defined on $6 \mathrm{~d}$ field $B_{i j}\left(x^{\mu}\right)$ is, in general, non-degenerate and the gauge condition $B_{i 6}=0$ does not lead to a non-trivial ( $A$-dependent) ghost determinant (cf. (2.11)). Note that the gauge-fixed action (2.14) is still invariant under the following 5 d local gauge transformations $\left(U\left(x^{i}\right) \in G\right)$ :

$$
B_{i j}^{\prime}=U B_{i j} U^{-1}, \quad A_{i}^{\prime}=U A_{i} U^{-1}+U \partial_{i} U^{-1}
$$

Provided the regularization preserves this symmetry, the effective action (2.16) should thus be built out of gauge-invariant combinations of $F_{i j}$ and $D_{i}$.

\section{$2.3 \quad$ Self-dual $B$-field model}

Let us now consider the analog of the non-abelian action (2.14) in the case of the $B$-field with a self-dual field strength. Let us first review the free-field case of a single self-dual field. In Minkowski signature the real 6d self-duality condition reads

$$
H_{\mu \nu \lambda}=\frac{1}{6} \epsilon_{\mu \nu \lambda \sigma \rho \delta} H^{\sigma \rho \delta} .
$$

As is well known, one way to find the action corresponding to (2.18) is to relax the manifest Lorentz symmetry. A systematic approach is to start with the phase-space path integral for the non-chiral $H_{\mu \nu \lambda}^{2}$ theory, impose the standard "time-like" gauge $B_{i 0}=0$, trade the momenta corresponding to $B_{i j}$ for another 2-form field and then impose the self-duality truncation ending up with the " $\mathcal{E B}-\mathcal{B B}$ " type action $(\mathcal{E}$ is "electric" and $\mathcal{B}$ is "magnetic") [17] (see also [18-21]). ${ }^{9}$ Switching to the Euclidean notation that we shall use below $\left(x^{0} \rightarrow i x^{6}\right.$, with the gauge condition $\left.B_{i 6}=0\right)$ the resulting action is $(i, j, \ldots=1, \ldots, 5)$

$$
\tilde{S}_{+}=\int d^{6} x \frac{1}{2} i \epsilon_{i j k p q} \partial_{k} B_{p q}\left(\partial_{6} B_{i j}+\frac{1}{2} i \epsilon_{i j r m n} \partial_{r} B_{m n}\right) .
$$

It formally has a residual gauge invariance $\delta B_{i j}=\partial_{i} \xi_{j}-\partial_{j} \xi_{i} \cdot{ }^{10}$ Taking the variation of (2.19) over $B_{i j}$ we obtain the equation of motion which may be written as ${ }^{11}$

$$
\partial_{[k} \mathcal{O}_{+} B_{i j]}=0, \quad\left(\mathcal{O}_{ \pm}\right)_{i j, m n} \equiv \delta_{i j, m n} \partial_{6} \pm \frac{1}{2} i \epsilon_{i j r m n} \partial_{r}
$$

\footnotetext{
${ }^{8}$ We ignore the $A_{i}$-independent factors in the partition function $Z=e^{-\Gamma}$ that should agree with (A.4) in the free limit. See appendix A for a discussion of the free $B$-field partition function in the "axial" gauge $B_{i 6}=0$.

${ }^{9}$ To get the right count of degrees of freedom at the level of path integral one should also keep track of appropriate Jacobians in the path integral measure.

${ }^{10}$ This a $6 \mathrm{~d}$ symmetry; the action is invariant up to a surface term. This residual symmetry is an artifact of the action $(2.19)$ - it is absent in the required self-duality equation $\mathcal{O}_{+} B_{i j}=0$.

${ }^{11}$ As in $(2.15)$, we adopt the standard convention $\delta_{i j}^{m n}=\frac{1}{2}\left(\delta_{i}^{m} \delta_{j}^{n}-\delta_{i}^{n} \delta_{j}^{m}\right)$.
} 
It is solved by

$$
\mathcal{O}_{+} B_{i j}=\partial_{i} q_{j}\left(x_{i}\right)-\partial_{j} q_{i}\left(x_{i}\right)+f_{i j}\left(x^{6}\right)
$$

for a $5 \mathrm{~d}$ function $q_{i}\left(x_{i}\right)$ and a function $f_{i j}\left(x^{6}\right)$ that does not depend on $5 \mathrm{~d}$ coordinates. Absorbing $q_{i}$ part into a formal redefinition of $B_{i j}$ in $\partial_{6} B_{i j}$ term in $\mathcal{O}_{+} B_{i j}$ and imposing the boundary condition that the self-duality condition $\mathcal{O}_{+} B_{i j}=0$ is satisfied at "spatial infinity" $\left|x^{i}\right|=\infty$ we conclude that $f_{i j}=0$ and thus $\mathcal{O}_{+} B_{i j}=0$ is satisfied everywhere. Integrating over $B_{i j}$ in the path integral defined by the action (2.19) and taking into account the necessary determinant factors in the measure one finds that the resulting partition function is

$$
Z_{+}=\left(\operatorname{det} \mathcal{O}_{+}^{\perp}\right)^{-1 / 2},
$$

where $\mathcal{O}_{+}^{\perp}$ acts on transverse $B_{i j}^{\perp}$ field and thus describes 3 dynamical degrees of freedom as expected. ${ }^{12}$

The equivalent results can be obtained by starting with an alternative $($ ( $\mathcal{E B}-\mathcal{E} \mathcal{E} ")$ action

$$
S_{+}=\int d^{6} x \partial_{6} B_{i j}\left(\partial_{6} B_{i j}+\frac{1}{2} i \epsilon_{i j k m n} \partial_{k} B_{m n}\right) .
$$

Here the equations of motion $\partial_{6}\left(\mathcal{O}_{+} B_{i j}\right)=0$ reduce to $\mathcal{O}_{+} B_{i j}=f_{i j}\left(x^{k}\right)$, and thus if the self-duality condition $\mathcal{O}_{+} B_{i j}=0$ is imposed at $\left|x^{6}\right|=\infty$, it is satisfied everywhere. The action (2.23) has a $5 \mathrm{~d}$ residual gauge symmetry $\delta B_{i j}=\partial_{i} \xi_{j}-\partial_{j} \xi_{i}$, where $\xi_{i}=\xi_{i}\left(x^{k}\right)$. The $B_{i j}$ path integral measure here should have an extra factor of $\left(\operatorname{det} \partial_{6}\right)^{1 / 2}$ that ensures 6d Lorentz invariance; as a result one finds the same chiral partition function (2.22) (cf. appendix A).

Note that the free limit of the non-chiral action (2.14) is equivalent to a combination of the self-dual and anti self-dual models: in the free limit the kinetic term in (2.14), (2.15) may be written as

$$
\left(\partial_{6} B_{i j}\right)^{2}+\frac{1}{3} H^{i j k} H_{i j k}=\mathcal{O}_{+} B_{i j} \mathcal{O}_{-} B_{i j},
$$

and thus the corresponding partition function is given by

$$
Z=\left(\operatorname{det} \Delta_{\perp}\right)^{-1 / 2}=Z_{+} Z_{-} .
$$

The above discussion has a straightforward generalization to the non-abelian case. Namely, let us require that the self-duality condition (2.18) or its Euclidean counterpart in the $B_{i 6}=0$ gauge should be satisfied for the non-abelian field strength (2.6) or (2.10), i.e.

$$
\hat{\mathcal{O}}_{+} B_{i j}=0, \quad\left(\hat{\mathcal{O}}_{ \pm}\right)_{i j, m n} \equiv \delta_{i j, m n} \partial_{6} \pm \frac{1}{2} i \epsilon_{i j k m n} D_{k}(A) .
$$

\footnotetext{
${ }^{12} B_{i j}^{\perp}$ has $\frac{1}{2} \times 4 \times 5-(5-1)=6$ real components and that the differential operator is a 1 -st order one (cf. appendix A).
} 
One may expect that this condition should follow (under the corresponding boundary conditions discussed above) from the direct analogs of (2.23) and (2.19):

$$
\begin{aligned}
& S_{+}=\int d^{6} x \operatorname{Tr}\left[\partial_{6} B_{i j}\left(\partial_{6} B_{i j}+\frac{1}{2} i \epsilon_{i j k m n} D_{k} B_{m n}\right)\right], \\
& \tilde{S}_{+}=\int d^{6} x \operatorname{Tr}\left[\frac{1}{2} i \epsilon_{i j r p q} D_{r} B_{p q}\left(\partial_{6} B_{i j}+\frac{1}{2} i \epsilon_{i j k m n} D_{k} B_{m n}\right)\right] .
\end{aligned}
$$

This is indeed obvious for (2.27) but is not immediately so for the second action (2.28). The equations of motion following from (2.28), $D_{[i} \hat{\mathcal{O}}_{+} B_{j k]}=0$, may be solved as $\hat{\mathcal{O}}_{+} B_{i j}=$ $q\left(x^{6}\right) F_{i j}\left(x^{k}\right)+f_{i j}\left(x^{6}\right)$, where $F_{i j}$ is the field strength of $A_{i}$. One may then attempt to absorb the $F$-term by a (non-local in $x^{6}$ ) redefinition $B_{i j} \rightarrow B_{i j}+\left(\partial_{6}\right)^{-1} q\left(x^{6}\right) F_{i j}\left(x^{k}\right)$ to arrive at the self-duality condition. However, the quantum equivalence of the theories based on (2.27) and (2.28) becomes unclear as an extra determinant of the operator $\frac{1}{2} i \epsilon_{i j r p q} D_{r}$ coming from (2.28) will now have a non-trivial dependence on $A_{i}$.

In what follows we shall use the simplest action (2.27) as defining the non-abelian selfdual $B$-field model. ${ }^{13}$ Since the $\partial_{6}$ operator factorizes in (2.27), the corresponding partition function is given by the direct analog of $(2.22)$ with $\mathcal{O}_{+} \rightarrow \hat{\mathcal{O}}_{+}(A) \cdot{ }^{14}$ It is straightforward to check that the operator $\Delta(A)$ in the non-chiral action $(2.14),(2.15)$ is given again by the product of the self-dual and anti self-dual operators in (2.26):

$$
\Delta_{i j}^{m n}(A)=-\hat{\mathcal{O}}_{+p q}^{m n}(A) \hat{\mathcal{O}}_{-i j}^{p q}(A) .
$$

As a result, the non-chiral $B$-field quantum effective action (2.16) may be written as

$$
\Gamma=\Gamma_{+}+\Gamma_{-}, \quad \Gamma_{ \pm}=\frac{1}{2} \log \operatorname{det} \hat{\mathcal{O}}_{ \pm}(A) .
$$

While $\Gamma$ should be parity $(\mathrm{P})$ even, $\Gamma_{+}$and $\Gamma_{-}$may a priori contain imaginary P-odd parts that cancel in their sum in (2.26) (as, e.g., in the case of an external gravitational field [14]). However, it is easy to see that this does not happen in the present case when the external field $A_{i}$ does not depend on $x^{6}$. Indeed, $\partial_{6} \rightarrow-\partial_{6}$ combined with $\epsilon_{5} \rightarrow-\epsilon_{5}$ is a symmetry of the classical action (2.27) and thus should be present also in the effective action. As the P-odd part of $\Gamma_{ \pm}$should contain an odd number of $\epsilon_{5}=\left(\epsilon_{i j k m n}\right)$ factors it should thus have an odd number of $p_{6}$ factors (in momentum representation) but then the integral over $p_{6}$ vanishes. The absence of P-odd part implies also the absence of an anomalous (5d gauge symmetry breaking) part of $\Gamma_{ \pm}$. Thus both the effective action $\Gamma$ of the full non-chiral theory and $\Gamma_{+}$of the self-dual theory should be invariant under the residual gauge symmetry of the $A$-field in (2.17).

To conclude, we have

$$
\Gamma=2 \Gamma_{+}, \quad \Gamma_{+}=\Gamma_{-}=\frac{1}{2} \log \operatorname{det} \hat{\mathcal{O}}_{+}(A) .
$$

\footnotetext{
${ }^{13}$ Like the free action (2.23) the interacting action (2.27) still has the residual $5 \mathrm{~d}$ gauge symmetry $\delta B_{i j}=\partial_{i} \xi_{j}-\partial_{j} \xi_{i}, \xi_{i}=\xi_{i}\left(x^{k}, 0\right)$ under which the variation of (2.27) is a total derivative: $\delta S_{+}=$ $\operatorname{Tr} \int d^{6} x \partial_{6} B_{i j}\left(i \epsilon_{i j k m n}\left[A_{k}, \partial_{m} \xi_{n}\right]\right)=\operatorname{Tr} \int d^{6} x \partial_{6}\left(B_{i j} i \epsilon_{i j k m n}\left[A_{k}, \partial_{m} \xi_{n}\right]\right)$. Since the parameter does not depend on $x^{6}$, this does not imply a degeneracy of the resulting kinetic operator for generic values of 6 -momentum and thus does not require gauge fixing.

${ }^{14}$ As in (2.16) we shall ignore constant $A$-independent factors in $Z$ : the operator $\hat{\mathcal{O}}_{+}$is acting on the full $B_{i j}$ rather than on its transverse part as in the free case in (2.22).
} 


\section{Structure of divergent part of effective action}

Before describing the details of the computation of the divergent part $\Gamma_{\infty}$ of the effective action (2.16) corresponding to non-chiral non-abelian $B$-field action (2.14) and the self-dual model (2.27) and verifying their relation in (2.31), let us first discuss the general structure of $\Gamma_{\infty}$ in a background gauge vector field.

Let us consider the 1-loop effective action for a $6 \mathrm{~d}$ model containing standard 2derivative Yang-Mills vectors, scalars and spinors coupled to a background gauge field. To prepare for the discussion of the models in the previous section we will specify to the case when the background field is chosen to be the 5-dimensional one as in (2.8) (i.e. use indices $m, n, \ldots=1, \ldots, 5)$. Using, e.g., the heat kernel representation and proper-time cutoff $\epsilon=\Lambda^{-2} \rightarrow 0$ one finds [12] from the general expression for the corresponding heat kernel coefficient [22] (see also [23, 24])

$$
\begin{aligned}
\Gamma_{\infty} & =-B_{6} \log \Lambda \\
B_{6} & =\frac{1}{(4 \pi)^{3}} \int d^{6} x\left[-\frac{1}{60} \beta_{2} \operatorname{tr}\left(D_{m} F_{m n} D_{k} F_{k n}\right)+\frac{1}{90} \beta_{3} \operatorname{tr}\left(F_{m n} F_{n k} F_{k m}\right)\right] .
\end{aligned}
$$

$\beta_{2}$ and $\beta_{3}$ are numerical coefficients of the two independent dimension- 6 invariants built out of the background field. ${ }^{15}$ Note that in dimensional regularization one gets $\Gamma_{\infty}=\frac{1}{\mathrm{~d}-6} B_{6}$ where $\frac{1}{\mathrm{~d}-6}$ corresponds to $-\log \Lambda$ in (3.1). Here tr is over the matrix indices of the particular representation to which the quantum field belongs: if it is in the adjoint representation (with hermitian generators $\left(t^{a}\right)_{b c}=-i f_{b c}^{a}$ ) one has the gauge field as a matrix $A_{n}^{a b}=f^{a c b} A_{n}^{c}$ and

$$
\begin{aligned}
\operatorname{tr}\left(D_{m} F_{m n} D_{k} F_{k n}\right) & =-C_{2} D_{m} F_{m n}^{a} D_{k} F_{k n}^{a}, \quad f_{a c d} f_{b c d}=C_{2} \delta_{a b} \\
\operatorname{tr}\left(F_{m n} F_{n k} F_{k m}\right) & =-\frac{1}{2} C_{2} f^{a b c} F_{m n}^{a} F_{n k}^{b} F_{k m}^{c} .
\end{aligned}
$$

For generic representation $R$ with generators satisfying $\operatorname{tr}\left(t^{a} t^{b}\right)=T_{R} \delta^{a b}$ one is to replace $C_{2}$ in (3.3), (3.4) by $T_{R}$.

For a collection of $N_{1} 6 \mathrm{~d}$ YM vectors, $N_{0}$ real scalars and $N_{\frac{1}{2}}$ Weyl fermions, each in adjoint representation, one finds $[12]^{16}$

$$
\beta_{2}=-36 N_{1}+N_{0}+16 N_{\frac{1}{2}}, \quad \quad \beta_{3}=4 N_{1}+N_{0}-4 N_{\frac{1}{2}} .
$$

Both coefficients vanish in the case of the maximally $(1,1)$ supersymmetric YM theory (SYM) in 6d which can be obtained by dimensional reduction from the 10d SYM giving $N_{1}=1, N_{0}=4, N_{\frac{1}{2}}=2 .{ }^{17}$ Note that the expression for the coefficient $\beta_{3}$ of the $F^{3}$ invariant in (3.5) happens to coincide with the number of effective degrees of freedom

\footnotetext{
${ }^{15}$ The other two invariants of the same dimension are related by use of Bianchi identities: $\operatorname{tr}\left(D_{m} F_{k n} D_{m} F_{k n}\right)=2 \operatorname{tr}\left(D_{m} F_{m n} D_{k} F_{k n}\right)-4 \operatorname{tr}\left(F_{m n} F_{n k} F_{k m}\right)+$ total derivative, $\operatorname{tr}\left(D_{m} F_{k n} D_{k} F_{m n}\right)=$ $\frac{1}{2} \operatorname{tr}\left(D_{m} F_{k n} D_{m} F_{k n}\right)$.

${ }^{16}$ We use this opportunity to correct two unfortunate misprints in [12]: $d-\frac{1}{2} \rightarrow d-42$ in eq. (3.9) (here $d=6)$ and $-\frac{1}{72} \rightarrow+\frac{1}{90}$ in eq. (3.5) (results in eq. (3.6) there are correct).

${ }^{17}$ Equivalently, if we consider the $(1,0)$ SYM coupled to one adjoint hypermultiplet we get the same 1-loop finite theory (cf. [27-29]).
} 
and so it vanishes also in the case of $(1,0) 6 \mathrm{~d}$ SYM where $N_{1}=1, N_{0}=0, N_{\frac{1}{2}}=1$. This is consistent with the fact that the only possible $(1,0) 6 \mathrm{~d}$ super-invariant is the one with the bosonic part containing $\left(D_{m} F_{m n}\right)^{2}$, i.e. the $F^{3}$ invariant is ruled out by $(1,0)$ supersymmetry (see $[25,26]$ ).

As we shall find below, in the case of the self-dual B-field the divergent part of the effective action $\Gamma_{+}$in (2.30) is given by (3.1), (3.2) with $\beta_{2}=-27, \beta_{3}=-57$. In the case of the non-chiral $B$-field with the effective action in (2.16) these coefficients are doubled, in agreement with (2.31). Thus, in the presence of $N_{T}$ self-dual tensors, the coefficients in (3.5) become

$$
\begin{aligned}
& \beta_{2}=-27 N_{T}-36 N_{1}+N_{0}+16 N_{\frac{1}{2}}, \\
& \beta_{3}=-57 N_{T}+4 N_{1}+N_{0}-4 N_{\frac{1}{2}} .
\end{aligned}
$$

Here all fields are assumed to be in the adjoint representation; otherwise $N_{T}, N_{0}, N_{\frac{1}{2}}$ are to be rescaled by the corresponding factors $T_{R} / C_{2}$.

\section{Calculation of one-loop divergences}

Let us now compute the coefficients in the logarithmically divergent part of the one-loop effective actions $\Gamma_{+}$and $\Gamma$ for the self-dual (2.27) and the non-chiral (2.14) 2-form models.

We adopt dimensional regularization, by continuing the theory to $\mathrm{d}=6-2 \varepsilon$ dimensions. Since the sixth direction is treated separately in the classical action and in the gauge fixing condition $\left(B_{i 6}=0\right)$, it is natural to keep it one-dimensional, while continuing the remaining 5 directions, setting $6=1+5 \rightarrow 1+d, d=5-2 \varepsilon$. Within the dimensional regularization we consider an analog of the four-dimensional helicity scheme, where all the momentum numerator algebra is first done in an integer number of dimensions and then the scalar integrals are continued to $d$ dimensions. This guarantees that the number of physical states in loops is unchanged by the regulator.

To find the coefficients in the divergent part of the effective action one may compute, e.g., the terms quadratic and cubic in the vector field $A$ and compare them with (3.2). This is what we will do below. Alternatively, one may find the terms with six powers of $A$ which appear in (3.2) without derivatives and thus can be isolated by taking the non-abelian field $A$ to be constant. This will be done in appendix $\mathrm{C}$ on the example of the self-dual model (2.27).

\subsection{Self-dual $B$-field model}

The effective action corresponding to the classical action $(2.27)$ is ${ }^{18}$

$$
\Gamma_{+}=\frac{1}{2} \log \operatorname{det} \Delta_{+}, \quad \Delta_{+} B_{i j}=-\partial_{6} \hat{\mathcal{O}}_{+} B_{i j}=-\partial_{6}\left(\partial_{6} B_{i j}+\frac{i}{2} \epsilon_{i j k m n} D_{k} B_{m n}\right) .
$$

\footnotetext{
${ }^{18}$ Compared to $(2.30),(2.31)$ here we include the $A$-independent factor $\partial_{6}$ in the kinetic operator making it symmetric.
} 
The operator $\Delta_{+}$is thus linear in the background field $A_{i}$, i.e. ${ }^{19}$

$$
\begin{aligned}
\Delta_{+} & =\Delta^{(0)}+\Delta^{(1)} \\
{\left[\Delta^{(0)}\right]_{i j, m n}^{a b} } & =-\delta^{a b}\left(\delta_{i j, m n} \partial_{6}^{2}+\frac{i}{2} \epsilon_{i j k m n} \partial_{6} \partial_{k}\right), \quad\left[\Delta^{(1)}\right]_{i j, m n}^{a b}=-\frac{i}{2} f^{a c b} \epsilon_{i j k m n} A_{k}^{c} \partial_{6} .
\end{aligned}
$$

Expanding the non-trivial part of $\Gamma_{+}$in powers of $A$, we have

$$
\begin{aligned}
\Gamma_{+}=\Gamma_{2}+\Gamma_{3}+\ldots, \quad \Gamma_{2} & =-\frac{1}{4} \operatorname{tr}\left[\left(\Delta^{(0)}\right)^{-1} \Delta^{(1)}\left(\Delta^{(0)}\right)^{-1} \Delta^{(1)}\right], \\
\Gamma_{3} & =\frac{1}{6} \operatorname{tr}\left[\left(\Delta^{(0)}\right)^{-1} \Delta^{(1)}\left(\Delta^{(0)}\right)^{-1} \Delta^{(1)}\left(\Delta^{(0)}\right)^{-1} \Delta^{(1)}\right] .
\end{aligned}
$$

Since the background field $A_{i}$ is independent of $x_{6}$, the trace projects out all terms containing an odd number of $\partial_{6}$ factors and also produces an overall factor of length $L_{6}=\int d x_{6}$. As was already mentioned in section 2 , together with the symmetry of the gauge-fixed action (2.27) under $\partial_{6} \rightarrow-\partial_{6}$ combined with $\epsilon_{5} \rightarrow-\epsilon_{5}$, this implies the effective action $\Gamma_{+}$is parity-even.

The evaluation of traces is standard, by using momentum space basis of states and assuming that the background field is $A_{i}^{a}\left(x_{k}\right)=\int \frac{d^{5} s}{(2 \pi)^{5}} \tilde{A}_{i}^{a}(s) e^{i s_{k} x_{k}}$. The matrix element of $\left(\Delta^{(0)}\right)^{-1}$ in momentum representation is the free $B$-field propagator

$$
\begin{aligned}
& \left\langle p\left|\left(\Delta^{(0)}\right)^{-1}\right| p\right\rangle \rightarrow \delta^{a b} P_{m n}^{j k}\left(p_{i}, p_{6}\right), \\
& P_{m n}^{j k}\left(p_{i}, p_{6}\right) \equiv \frac{1}{\left(p_{i}^{2}+p_{6}^{2}\right)}\left(\delta_{m n}^{j k}-\frac{i}{2} \frac{\epsilon_{m n q}{ }^{j k} p_{q}}{p_{6}}+2 \frac{p^{[j} p_{[m} \delta_{n]}^{k]}}{p_{6}^{2}}\right) .
\end{aligned}
$$

The matrix element of $\Delta^{(1)}$ is the vertex

$$
\left\langle p+s\left|\Delta^{(1)}\right| p\right\rangle \rightarrow V_{i j}^{a b m n}\left(s_{i}, p_{6}\right) \equiv \frac{1}{2} f^{a c b} \epsilon_{i j}{ }^{k m n} p_{6} \tilde{A}_{k}^{c}\left(s_{i}\right) .
$$

\subsection{1 $A^{2}$ term}

Inserting complete sets of momentum eigenstates between any two operators in (4.4) and using (4.5), (4.6) and momentum conservation, we have ${ }^{20}$

$$
\begin{aligned}
\Gamma_{2} & =L_{6} \int \frac{d^{5} s}{(2 \pi)^{5}} \mathcal{G}_{2}(s), \\
\mathcal{G}_{2}(s) & =-\frac{1}{4} \int \frac{d p_{6} d^{d} p}{(2 \pi)^{d+1}} V_{i_{1} i_{2}}^{c d} j_{1} j_{2}\left(s_{i}, p_{6}\right) P_{j_{1} j_{2}}^{k_{1} k_{2}}\left(p_{i}, p_{6}\right) V_{k_{1} k_{2}}^{d c} l_{1} l_{2}\left(-s_{i}, p_{6}\right) P_{l_{1} l_{2}}^{i_{1} i_{2}}\left(p_{i}+s_{i}, p_{6}\right),
\end{aligned}
$$

where $d=5-2 \epsilon$ and $L_{6}=\int d x_{6}$. Since the external field does not depend on $x_{6}$ here all the factors have the same 6 -th component of momentum $p_{6}$.

\footnotetext{
${ }^{19}$ Here $a, b, c$ are Lie algebra indices. We assume that $B$ is in adjoint representation; otherwise $t_{b c}^{a}=-i f_{b c}^{a}$ is to be replaced by the corresponding hermitian generators.

${ }^{20}$ The same expression may be obtained by computing the two-point function of $A$ and promoting it to a term in the effective action. In this approach, the numerical factors are symmetry factors and the signs related to resummation of one-loop corrections to the $A$-field two-point function.
} 
The background-field gauge invariance requires that (4.7) should vanish for constant $A_{i}$, i.e. for $s_{i}=0$. Setting $s_{i}=0$ and carrying out index contractions we get

$$
\mathcal{G}_{2} \propto \int \frac{d p_{6} d^{d} p}{(2 \pi)^{d+1}} \frac{3 p_{i}^{2}+5 p_{6}^{2}}{\left(p_{i}^{2}+p_{6}^{2}\right)^{2}}=\frac{d-5}{d-2} \int \frac{d p_{6} d^{d} p}{(2 \pi)^{d+1}} \frac{2 p_{6}^{2}}{\left(p_{i}^{2}+p_{6}^{2}\right)^{2}}
$$

where we used the identity (B.4). Thus, for a constant external field, the $A^{2}$ contribution vanishes in $d=5$ even before performing the integration over the $p_{6}$ momentum.

Contracting group indices using eq. (3.3), introducing Feynman parameter $y$ in the momentum integral, doing tensor reduction with the help of (B.2), and finally using the identity (B.4) gives the following expression for (4.7):

$$
\begin{aligned}
\Gamma_{2} & =\frac{1}{4} C_{2} L_{6} \int \frac{d^{5} s}{(2 \pi)^{5}} \tilde{A}_{i}^{a}(s)\left(\delta_{i j} s^{2}-s_{i} s_{j}\right) \Pi\left(s^{2}\right) \tilde{A}_{j}^{a}(-s), \\
\Pi\left(s^{2}\right) & =\int_{0}^{1} d y \int \frac{d p_{6} d^{d} p}{(2 \pi)^{d+1}} \frac{(1-y)\left[(1-12 y) p_{6}^{2}-2 y s^{2}\right]}{2 p_{6}^{2}\left[p_{i}^{2}+p_{6}^{2}+y(1-y) s^{2}\right]^{2}} .
\end{aligned}
$$

The $d$-dimensional integral here is standard (cf. eq. (B.1)); while it is finite for $d \rightarrow 5$, taking this limit before the $p_{6}$ integral makes the latter divergent. To carry out the $p_{6}$ integral it is convenient to change the variable $p_{6} \rightarrow \mu$ as $p_{6}=\mu\left[y(1-y) s^{2}\right]^{1 / 2}$; then the remaining $\mu$-integral can be computed using (B.5). As a result, we find that the divergent part of the $A^{2}$ term in the effective action is

$$
\Gamma_{2 \infty}=\frac{1}{d-5} \frac{9 C_{2}}{5 \times 2^{8} \pi^{3}} L_{6} \int \frac{d^{5} s}{(2 \pi)^{5}} \tilde{A}_{i}^{a}(s) s^{2}\left(s^{i} s^{j}-\delta^{i j} s^{2}\right) \tilde{A}_{j}^{a}(-s)
$$

Comparing this with the first term in (3.2), (3.3) (with $\frac{1}{d-5}$ identified with $-\log \Lambda$ in (3.1)) we conclude that (cf. (3.6))

$$
\beta_{2}=-27
$$

As was already mentioned below (3.4), in the case of the $B$-field being in generic representation $R$ the coefficient $C_{2}$ is to be replaced by the corresponding index $T_{R}$.

\subsection{2 $\quad A^{3}$ term}

To find $\beta_{3}$ in (3.2) we need to compute the $A^{3}$ part of the effective action. The evaluation of $\Gamma_{3}$ in (4.4) follows the same steps as that of $\Gamma_{2}$. For a $B$-field in an arbitrary representation (4.4) becomes

$$
\begin{aligned}
\Gamma_{3}=L_{6} \int \frac{d^{5} s_{1}}{(2 \pi)^{5}} \frac{d^{5} s_{2}}{(2 \pi)^{5}} \frac{d^{5} s_{3}}{(2 \pi)^{5}} \mathcal{G}_{3}\left(s_{1}, s_{2}, s_{3}\right) \delta^{(5)}\left(s_{1}+s_{2}+s_{3}\right) \\
\mathcal{G}_{3}=\frac{1}{6} \int \frac{d p_{6} d^{d} p}{(2 \pi)^{d+1}} \operatorname{tr}\left[V_{j_{5} j_{6}}^{i_{1} i_{2}}\left(s_{1 i}, p_{6}\right) P_{i_{1} i_{2}}^{j_{1} j_{2}}\left(p_{i}, p_{6}\right) V_{j_{1} j_{2}}^{i_{3} i_{4}}\left(s_{2 i}, p_{6}\right)\right. \\
\left.\times P_{i_{3} i_{4}}^{j_{3} j_{4}}\left(p_{i}+s_{2 i}, p_{6}\right) V_{j_{3} j_{4}}^{i_{5} i_{6}}\left(s_{3 i}, p_{6}\right) P_{i_{5} i_{6}}^{j_{5} j_{6}}\left(p_{i}+s_{2 i}+s_{3 i}, p_{6}\right)\right] .
\end{aligned}
$$


Here $V_{m n}^{i j}$ is the vertex in (4.6) with $f^{a c b}$ replaced by $-i t^{c}$ where $t^{c}$ is hermitian generator in some representation $R$ (coming from the covariant derivative $D_{i} B=\partial_{i} B-i t^{a} A_{i}^{a} B$ ). To compute the trace over the group indices we use that

$$
\operatorname{tr}\left(t^{a} t^{b} t^{c}\right)=\frac{1}{2} T_{R} f^{a b c}+\frac{1}{2} \mathrm{~A}_{R} d^{a b c},
$$

where $\mathrm{A}_{R}$ is the anomaly coefficient of a given representation. In adjoint representation $T_{R}=C_{2}, \mathrm{~A}_{R}=0$. The momentum-dependent coefficient of the symmetric $d^{a b c}$ tensor part is P-odd (containing one power of $\epsilon_{5}$ ) and should thus vanish identically as discussed above.

After carrying out the index contraction, Feynman parametrization and momentum integration, the divergent part of $\mathcal{G}_{3}$ may be written as (in the adjoint representation)

$$
\mathcal{G}_{3 \infty}=\frac{1}{d-5} \frac{i}{15 \times 2^{8} \pi^{3}} C_{2} f^{a_{1} a_{2} a_{3}} K^{a_{1} a_{2} a_{3}}\left(s_{1}, s_{2}, s_{3}\right),
$$

where $K^{a_{1} a_{2} a_{3}}$ is a $5 \mathrm{~d}$ invariant constructed from 3 powers of the background field $\tilde{A}_{i}\left(s_{k}\right)$ and the corresponding momenta $\left(s_{r}^{2} \equiv s_{r} \cdot s_{r}\right)$ :

$$
\begin{aligned}
& K^{a_{1} a_{2} a_{3}}=\tilde{A}^{a_{1}}\left(s_{1}\right) \cdot s_{1}\left[-9 \tilde{A}^{a_{2}}\left(s_{2}\right) \cdot s_{1}\left(2 \tilde{A}^{a_{3}}\left(s_{3}\right) \cdot s_{1}+\tilde{A}^{a_{3}}\left(s_{3}\right) \cdot s_{3}\right)\right. \\
& \left.+\tilde{A}^{a_{2}}\left(s_{2}\right) \cdot s_{3}\left(\tilde{A}^{a_{3}}\left(s_{3}\right) \cdot s_{1}+9 \tilde{A}^{a_{3}}\left(s_{3}\right) \cdot s_{3}\right)\right] \\
& +\tilde{A}^{a_{1}}\left(s_{1}\right) \cdot s_{3}\left[-\tilde{A}^{a_{2}}\left(s_{2}\right) \cdot s_{1}\left(19 \tilde{A}^{a_{3}}\left(s_{3}\right) \cdot s_{1}+\tilde{A}^{a_{3}}\left(s_{3}\right) \cdot s_{3}\right)\right. \\
& \left.+\tilde{A}^{a_{2}}\left(s_{2}\right) \cdot s_{3}\left(19 \tilde{A}^{a_{3}}\left(s_{3}\right) \cdot s_{1}+18 \tilde{A}^{a_{3}}\left(s_{3}\right) \cdot s_{3}\right)\right] \\
& +\tilde{A}^{a_{1}}\left(s_{1}\right) \cdot \tilde{A}^{a_{2}}\left(s_{2}\right)\left[\tilde{A}^{a_{3}}\left(s_{3}\right) \cdot s_{1}\left(36 s_{1}^{2}+36 s_{2}^{2}-2 s_{3}^{2}\right)\right. \\
& \left.+\tilde{A}^{a_{3}}\left(s_{3}\right) \cdot s_{3}\left(17 s_{1}^{2}+19 s_{2}^{2}-s_{3}^{2}\right)\right] \\
& +\tilde{A}^{a_{1}}\left(s_{1}\right) \cdot \tilde{A}^{a_{3}}\left(s_{3}\right)\left[\tilde{A}^{a_{2}}\left(s_{2}\right) \cdot s_{1}\left(-19 s_{1}^{2}+s_{2}^{2}-17 s_{3}^{2}\right)\right. \\
& \left.+\tilde{A}^{a_{2}}\left(s_{2}\right) \cdot s_{3}\left(17 s_{1}^{2}-s_{2}^{2}+19 s_{3}^{2}\right)\right] \\
& +\tilde{A}^{a_{2}}\left(s_{2}\right) \cdot \tilde{A}^{a_{3}}\left(s_{3}\right)\left[\tilde{A}^{a_{1}}\left(s_{1}\right) \cdot s_{1}\left(s_{1}^{2}-19 s_{2}^{2}-17 s_{3}^{2}\right)\right. \\
& \left.+2 \tilde{A}^{a_{1}}\left(s_{1}\right) \cdot s_{3}\left(s_{1}^{2}-18 s_{2}^{2}-18 s_{3}^{2}\right)\right] .
\end{aligned}
$$

It simplifies in the transverse background gauge $s_{i} \tilde{A}_{i}^{a}(s)=0$ :

$$
\begin{aligned}
K^{a_{1} a_{2} a_{3}}= & -19 s_{3} \cdot \tilde{A}^{a_{1}}\left(s_{1}\right) s_{1} \cdot \tilde{A}^{a_{3}}\left(s_{3}\right)\left(s_{1}-s_{3}\right) \cdot \tilde{A}^{a_{2}}\left(s_{2}\right) \\
& +\left[18\left(s_{1}^{2}+s_{2}^{2}\right)-s_{3}^{2}\right] \tilde{A}^{a_{1}}\left(s_{1}\right) \cdot \tilde{A}^{a_{2}}\left(s_{2}\right) s_{1} \cdot \tilde{A}^{a_{3}}\left(s_{3}\right) \\
& +\left[18\left(s_{2}^{2}+s_{3}^{2}\right)-s_{1}^{2}\right] \tilde{A}^{a_{2}}\left(s_{2}\right) \cdot \tilde{A}^{a_{3}}\left(s_{3}\right) s_{2} \cdot \tilde{A}^{a_{1}}\left(s_{3}\right) \\
& +\left[18\left(s_{3}^{2}+s_{1}^{2}\right)-s_{2}^{2}\right] \tilde{A}^{a_{3}}\left(s_{3}\right) \cdot \tilde{A}^{a_{1}}\left(s_{1}\right) s_{3} \cdot \tilde{A}^{a_{2}}\left(s_{2}\right) .
\end{aligned}
$$

Comparing this to the two terms in (3.2) (which both contribute to the $A^{3}$ term) and using that $\beta_{2}$ was already determined in (4.12) we conclude that (cf. (3.7))

$$
\beta_{3}=-57 \text {. }
$$

We have confirmed this result independently by computing constant $A^{6}$ term in the effective action in appendix $\mathrm{C}$. 


\section{$4.2 \quad$ Non-chiral $B$-field model}

In the non-chiral theory (2.14), (2.15) the effective action (2.16) is given by

$$
\Gamma=\frac{1}{2} \ln \operatorname{det} \Delta, \quad \Delta B_{i j}=-\left(\partial_{6}^{2}+D^{2}\right) B_{i j}+2 \delta_{[i}^{[m} D^{n]} D_{j]} B_{m n} .
$$

Here $\Delta$ is quadratic in the background field $A_{i}$, i.e. (cf. (4.2), (4.3))

$$
\begin{aligned}
\Delta & =\Delta^{(0)}+\Delta^{(1)}+\Delta^{(2)} \\
{\left[\Delta^{(0)}\right]_{i j, m n}^{a c} } & =\delta^{a c}\left[-\delta_{i j, m n}\left(\partial_{i}^{2}+\partial_{6}^{2}\right)+2 \delta_{[m[i} \partial_{j]} \partial_{n]}\right] \\
{\left[\Delta^{(1)}\right]_{i j, m n}^{a c} } & =f^{a b c}\left[-\delta_{i j, m n}\left(\partial_{k} A_{k}^{b}+2 A_{k}^{b} \partial_{k}\right)+2 \delta_{[i[m}\left(A_{n]}^{b} \partial_{j]}+\partial_{n]} A_{j]}^{b}+A_{j]}^{b} \partial_{n]}\right)\right] \\
{\left[\Delta^{(2)}\right]_{i j, m n}^{a c} } & =f^{a d e} f^{e b c}\left[-\delta_{i j, m n} A_{k}^{d} A_{k}^{b}+2 \delta_{[i[m} A_{n]}^{d} A_{j]}^{b}\right]
\end{aligned}
$$

The quadratic and cubic in $A_{i}$ parts of the effective action have the structure (cf. (4.4))

$$
\begin{aligned}
\Gamma & =\Gamma_{2}+\Gamma_{3}+\ldots, \\
\Gamma_{2} & =\frac{1}{2} \operatorname{tr}\left[\left(\Delta^{(0)}\right)^{-1} \Delta^{(2)}\right]-\frac{1}{4} \operatorname{tr}\left[\left(\Delta^{(0)}\right)^{-1} \Delta^{(1)}\left(\Delta^{(0)}\right)^{-1} \Delta^{(1)}\right] \\
\Gamma_{3} & =-\frac{1}{2} \operatorname{tr}\left[\left(\Delta^{(0)}\right)^{-1} \Delta^{(2)}\left(\Delta^{(0)}\right)^{-1} \Delta^{(1)}\right]+\frac{1}{6} \operatorname{tr}\left[\left(\Delta^{(0)}\right)^{-1} \Delta^{(1)}\left(\Delta^{(0)}\right)^{-1} \Delta^{(1)}\left(\Delta^{(0)}\right)^{-1} \Delta^{(1)}\right] .
\end{aligned}
$$

The analogs of the relations (4.5), (4.6) in momentum representation are

$$
\begin{aligned}
\left\langle p\left|\left(\Delta^{(0)}\right)^{-1}\right| p\right\rangle & \rightarrow \delta^{a b} P_{m n}^{i j}\left(p_{k}, p_{6}\right), \quad P_{m n}^{i j}\left(p_{k}, p_{6}\right)=\frac{1}{\left(p_{i}^{2}+p_{6}^{2}\right)}\left(\delta_{m n}^{i j}+2 \frac{p^{[i} p_{[m} \delta_{n]}^{j]}}{p_{6}^{2}}\right), \\
\left\langle p+s\left|\Delta^{(1)}\right| p\right\rangle & \rightarrow V^{(1) a b m n}\left(p_{k}, s_{k}\right) \\
& =-i f^{a c b}\left[\delta_{i j}^{m n} \tilde{A}_{k}^{c}\left(s_{k}+2 p_{k}\right)+2 \delta_{[j}^{[m}\left(\tilde{A}_{i]}^{c} s^{n]}+\tilde{A}^{n] c} p_{i]}+\tilde{A}_{i]}^{c} p^{n]}\right)\right], \\
\left\langle p+s_{1}+s_{2}\left|\Delta^{(2)}\right| p\right\rangle & \rightarrow V_{i j}^{(2) a b m n}\left(p_{k}, s_{1 k}, s_{2 k}\right)=f^{a d e} f^{b c e}\left(\delta_{i j}^{m n} \tilde{A}_{k}^{d} \tilde{A}_{k}^{c}+2 \delta_{[j}^{[m} \tilde{A}^{n] d} \tilde{A}_{i]}^{c}\right) .
\end{aligned}
$$

\subsection{1 $\quad A^{2}$ term}

The first term in $\Gamma_{2}$ in (4.22) is a tadpole which vanishes in dimensional regularization; the second term gives (using the same notation as in (4.14))

$$
\begin{aligned}
& \Gamma_{2}=L_{6} \int \frac{d^{5} s}{(2 \pi)^{5}} \mathcal{G}_{2}(s), \\
& \mathcal{G}_{2}=-\frac{1}{4} \int \frac{d p_{6} d^{d} p}{(2 \pi)^{d+1}} V_{i_{1} i_{2}}^{(1) c j_{1} j_{2}}\left(s_{i}, p_{6}\right) P_{j_{1} j_{2}}^{k_{1} k_{2}}\left(p_{i}, p_{6}\right) \\
& \times V_{k_{1} k_{2}}^{(1) d c} l_{1} l_{2}\left(p_{i}+s_{i},-s_{i}\right) P_{l_{1} l_{2}}^{i_{1} i_{2}}\left(p_{i}+s_{i}, p_{6}\right) .
\end{aligned}
$$


Following similar steps as in the self-dual model in section 4.1 .1 we find $\left(p^{2}=p_{i} p_{i}\right)$

$$
\begin{aligned}
\mathcal{G}_{2}= & -\frac{3}{2} C_{2} \int_{0}^{1} d y \int \frac{d p_{6} d^{d} p}{(2 \pi)^{d+1}} Q\left(s_{i}, p_{k}, p_{6}, y\right) \\
Q= & \left(\left[\frac{1}{2}-y(1-y)\right] s^{2}+y^{2}(1-y)^{2} \frac{s^{4}}{p_{6}^{2}}\right. \\
& \left.\quad+\frac{8}{5} p^{2}+[5-26 y(1-y)] \frac{s^{2} p^{2}}{10 p_{6}^{2}}+\frac{12 p^{4}}{5 p_{6}^{2}}\right) \tilde{A}^{a}(s) \cdot \tilde{A}^{a}(-s) \\
& -\left[\frac{1}{2}-3 y(1-y)-y^{2}(1-y)^{2} \frac{s^{2}}{p_{6}^{2}}-[1-18 y(1-y)] \frac{p^{2}}{10 p_{6}^{2}}\right] s \cdot \tilde{A}^{a}(s) s \cdot \tilde{A}^{a}(-s) .
\end{aligned}
$$

It is useful to use eq. (B.4) to relate the integrals with loop momenta in the numerators to scalar bubble integrals. Unlike the self-dual theory case, here the $A^{2}$ term takes a gauge-invariant form only after one carries out all the integrals; its divergent part is found to be

$$
\Gamma_{2 \infty}=\frac{1}{d-5} \frac{9 C_{2}}{5 \times 2^{8} \pi^{3}} L_{6} \int \frac{d^{5} s}{(2 \pi)^{5}} \tilde{A}_{i}^{a}(s) s^{2}\left(s^{i} s^{j}-\delta^{i j} s^{2}\right) \tilde{A}_{j}^{a}(-s)
$$

This is twice the value in the self-dual case (4.11), i.e. the corresponding $\beta_{2}$ coefficient in (3.2) is (cf. (4.12))

$$
\beta_{2}=-54=2 \beta_{2}^{\text {self-dual }}
$$

\subsection{2 $\quad A^{3}$ term}

Unlike the case of $\Gamma_{2}$, the matrix element of $\Delta^{(2)}$ in (4.25) contributes nontrivially to $\Gamma_{3}$ in (4.22) (cf. (4.13))

$$
\begin{aligned}
& \Gamma_{3}=L_{6} \int \frac{d^{5} s_{1}}{(2 \pi)^{5}} \frac{d^{5} s_{2}}{(2 \pi)^{5}} \frac{d^{5} s_{3}}{(2 \pi)^{5}} \mathcal{G}_{3}\left(s_{1}, s_{2}, s_{3}\right) \delta^{(5)}\left(s_{1}+s_{2}+s_{3}\right), \\
& \mathcal{G}_{3}=\int \frac{d p_{6} d^{d} p}{(2 \pi)^{d+1}}\left[-\frac{1}{2} V_{i_{1} i_{2}}^{(2) c d j_{1} j_{2}}\left(p_{i}, s_{1 i}, s_{2 i}\right) P_{j_{1} j_{2}}^{k_{1} k_{2}}\left(p_{i}, p_{6}\right)\right. \\
& \times\left. V_{k_{1} k_{2}}^{(1) d c l_{1} l_{2}}\left(q_{i}, s_{3 i}\right) P_{l_{1} l_{2}}^{i_{1} i_{2}}\left(q_{i}, p_{6}\right)\right|_{q=p-s_{3}} \\
& +\frac{1}{6} V_{j_{5} j_{6}}^{(1) d e i_{1} i_{2}}\left(p_{i}, s_{2, i}\right) P_{i_{1} i_{2}}^{j_{1} j_{2}}\left(p_{i}, p_{6}\right) V_{j_{1} j_{2}}^{(1) e f i_{3} i_{4}}\left(q_{i}, s_{1 i}\right) \\
& \left.\times\left. P_{i_{3} i_{4}}^{j_{3} j_{4}}\left(q_{i}, p_{6}\right) V_{j_{3} j_{4}}^{(1) f i_{5} i_{6}}\left(r_{i}, s_{3 i}\right) P_{i_{5} i_{6}}^{j_{5} j_{6}}\left(r_{i}, p_{6}\right)\right|_{q=p-s_{1}, r=p-s_{1}-s_{3}}\right] .
\end{aligned}
$$

Here we used (4.15) to do the group index contraction (with the momentum-dependent coefficient of $d^{a b c}$ again vanishing in general) and considered the adjoint representation. Introducing Feynman parameters and shifting the integration variable in such a way that the denominator becomes a symmetric function, we use the $\mathrm{SO}(d)$ symmetry to express the tensor momentum integrals in terms of the scalar ones. Further using eq. (B.4) the integrals with various powers of the $d$-momentum can be reduced to scalar triangle and/or bubble integrals. Evaluating first the $d$-dimensional integral and then appropriately changing 
the variable of the $p_{6}$ integral one can decouple the latter from that over the Feynman parameters. As before, the logarithmic UV divergence we are interested in emerges after the last $\left(p_{6}\right)$ integral is evaluated using (B.5).

The contribution of the first structure in (4.33) written in coordinate representation gives a term proportional to $\frac{1}{d-5} L_{6} \int d^{5} x f^{a b c} A_{i}^{a} A_{j}^{b} \partial^{2}\left(\partial_{i} A_{j}^{c}-\partial_{j} A_{i}^{c}\right)$ which matches the cubic term in $\operatorname{tr}\left(\partial_{m} F_{m n}\right)^{2}$ in (3.2). Gauge invariance and consistency with the $A^{2}$ term calculation (4.30) are restored by the inclusion of the second term in (4.33).

The full computation is straightforward but tedious so we simply state that the final result is consistent with (3.2) with $\beta_{2}$ found above in (4.31) and with $\beta_{3}$ being again twice the value in the self-dual case (4.19):

$$
\beta_{3}=-114=2 \beta_{3}^{\text {self-dual }} .
$$

\section{Concluding remarks}

In this paper we have studied a model of $6 \mathrm{~d} 2$-form $B$-fields in some representation of an internal symmetry group $G$ coupled consistently to a non-abelian gauge field $A$ which "lives" only in a 5d subspace. We computed one-loop logarithmic divergences in such a theory by integrating out the $B$-field and treating the gauge field $A$ as a background. The resulting divergent part of the effective action (3.1), (3.2) contains the terms $\sim \operatorname{tr}\left[3 \beta_{2}\left(D_{m} F_{m n}\right)^{2}-2 \beta_{3} F_{m n} F_{n k} F_{k m}\right]$ with the coefficients $\beta_{2}, \beta_{3}$ given by (3.6), (3.7) or (4.12), (4.19) in the case of the self-dual theory (and twice these values in the case of the non-chiral $B$-field model given in (4.31), (4.34)).

The presence of these divergences suggests that in the full theory where $A$-field (or its $6 \mathrm{~d}$ extension) is also quantized the higher-derivative $c_{1}(D F)^{2}+c_{2} F^{3}$ terms should be added to the bare $6 \mathrm{~d}$ action. ${ }^{21}$ One may hope that these divergences could be cancelled by adding some other fields to the model and imposing, e.g., supersymmetry constraint. For a collection of $N_{T}$ self-dual tensors, $N_{1}$ standard 2-derivative YM vectors, $N_{0}$ real scalars and $N_{\frac{1}{2}}$ Weyl fermions in $6 \mathrm{~d}$ coupled to background vector, we conclude that ${ }^{22}$

$$
\beta_{2}=-27 N_{T}-36 N_{1}+N_{0}+16 N_{\frac{1}{2}}, \quad \quad \beta_{3}=-57 N_{T}+4 N_{1}+N_{0}-4 N_{\frac{1}{2}} .
$$

Thus the self-dual $B$-field coupled minimally to a 5 d gauge field contributes to the $\beta_{2}$ in the logarithmic divergent part of the effective action with the same sign as the YM vector. A somewhat unexpected feature is that its contribution to $\beta_{3}$ turns out to be opposite in sign compared to other standard 2-derivative bosonic fields. A naive expectation could be

\footnotetext{
${ }^{21}(D F)^{2}+F^{3}$ theory is of course classically conformally invariant but this symmetry will be anomalous at loop level. Let us note that the presence of similar $(D F)^{2}$ terms in $6 \mathrm{~d}$ theory was suggested by requiring dual conformal symmetry in six dimensions in [30], though precise connection of this to the present work is not clear to us.

${ }^{22}$ Note that quantizing the $5 \mathrm{~d}$ gauge field with the action $L_{6} \int d^{5} x\left[c_{1}\left(D_{i} F_{i j}\right)^{2}+c_{2} F_{i j}^{3}\right]$ will not produce extra one-loop logarithmic UV divergences as this theory is effectively 5 -dimensional one. It would be interesting to add also the one-loop contribution of the genuine $6 \mathrm{~d}$ non-abelian vector model. Choosing the background gauge field to be a $5 \mathrm{~d}$ one we would then get additional contributions to $\beta_{2}$ and $\beta_{3}$.
} 
that each field should contribute to $\beta_{3}$ proportionally to its number of dynamical degrees of freedom:

$$
\nu=3 N_{T}+4 N_{1}+N_{0}-4 N_{\frac{1}{2}} .
$$

One may formally consider fields that form $6 \mathrm{~d}$ supermultiplets containing self-dual $B$-field and couple them to a background $5 \mathrm{~d}$ gauge field. In the case of $(1,0)$ tensor multiplet with $N_{T}=1, N_{1}=0, N_{0}=1, N_{\frac{1}{2}}=1$ a natural coupling is to $(1,0) \operatorname{SYM}\left(N_{1}=\right.$ $\left.1, N_{0}=0, N_{\frac{1}{2}}=1\right)$; in this case we would expect to get $\beta_{3}=0$ as the $F^{3}$-invariant should be prohibited by supersymmetry. However, while $\nu$ in (5.2) indeed vanishes in this case, from (5.1) we get $\beta_{3}=-60=2 \beta_{2}$. This suggests that the model considered in this paper (with $A_{i}$ treated as a 5 d background field) does not admit a $(1,0)$ supersymmetric extension. This may not be surprising as the model lacks $6 \mathrm{~d}$ Lorentz symmetry. ${ }^{23}$

A possible role or application of the non-abelian coupled $(B, A)$ model discussed in this paper remains an open question. It might be related to some intersecting brane configuration where a $5 \mathrm{~d}$ gauge field lives on a $5 \mathrm{~d}$ brane "defect". Another option is that the $5 \mathrm{~d} A$-field may happen to play an auxiliary role and eliminating it one may end up with an effective interacting theory of a set of $B$-field only. Yet another possibility is the existence of a generalization where the $A$ gauge field becomes fully 6 -dimensional, Lorentz invariance is formally restored but the resulting classical action might become effectively non-local.

\section{Acknowledgments}

KWH would like to thank P.-M. Ho and E. Witten for discussions of the non-local gauge symmetry and remarks on M5-branes at an early stage of this work. AAT is grateful to R. Metsaev and K. Mkrtchyan for related discussions of $6 \mathrm{~d}$ theories. The work of KWH was supported in part by the National Science Foundation under Grant No. PHY-1620628. The work of RR was supported by the Department of Energy under Award Number DESC0013699. AAT was supported by the STFC grant ST/P000762/1 and by the Russian Science Foundation grant 14-42-00047 at Lebedev Institute.

\section{A Free partition function}

For a free rank 2 antisymmetric tensor $B_{\mu \nu}$ in $d$ dimensions with action $\int d^{d} x H_{\mu \nu \lambda} H^{\mu \nu \lambda}, H=d B$, the partition function in the covariant Feynman-like gauge (found by adding the $\left(\partial_{\mu} B_{\mu \nu}\right)^{2}$ term to the action) is $[32,33]$

$$
Z=\left[\frac{\left(\operatorname{det} \Delta_{1}\right)^{2}}{\operatorname{det} \Delta_{2}\left(\operatorname{det} \Delta_{0}\right)^{3}}\right]^{1 / 2},
$$

\footnotetext{
${ }^{23}$ If we naively consider the case of $(2,0)$ tensor multiplet with $N_{T}=1, N_{1}=0, N_{0}=5, N_{\frac{1}{2}}=2$, we obtain $\beta_{2}=-\frac{1}{6} \beta_{3}=10$. It is also interesting to note that similar $\int d^{6} x \operatorname{tr}\left[c_{1}\left(D_{\mu} F_{\mu \nu}\right)^{2}+c_{2} F_{\mu \nu} F_{\nu \lambda} F_{\lambda \mu}\right]$ divergences (or contributions to stress tensor anomaly) appear if one couples $(2,0)$ tensor multiplet to the R-symmetry $\mathrm{SO}(5)$ vector gauge field [31]; as the $B$-field is singlet under the $\mathrm{SO}(5)$, there the contribution comes only from the coupling of the $\mathrm{SO}(5)$ field to the scalars and fermions.
} 
where the free Laplacians $\Delta_{n}=-\partial^{2}$ are defined on rank $n$ antisymmetric tensors. The number of dynamical degrees of freedom $\nu_{2}(d)$ of rank 2 tensor in $d$ dimensions extracted from the representation of $Z$ as $\left[\operatorname{det} \Delta_{0}\right]^{-\nu / 2}$ is then

$$
\nu_{2}(d)=C_{d-2}^{2}=\frac{1}{2}(d-2)(d-3), \quad \nu_{2}(6)=6 .
$$

For a self-dual tensor in 6 dimensions we should get $\nu_{2,+}(6)=3$. Eq.(A.1) may also be written as

$$
Z=\left[\frac{\operatorname{det} \Delta_{1 \perp}}{\operatorname{det} \Delta_{2 \perp} \operatorname{det} \Delta_{0}}\right]^{1 / 2}
$$

where $\Delta_{n \perp}$ are defined on transverse tensors. ${ }^{24}$ The count of degrees of freedom in $6 \mathrm{~d}$ goes as follows: $\nu_{2}(6)=(15-5)+1-(6-1)=6\left(\partial_{\mu} B_{\mu \nu}=0\right.$ gives $6-1=5$ conditions, etc. $)$.

The equivalent results are found also in the "axial" gauge $B_{i 6}=0(i=1, \ldots, 5)$ where $H_{6 i j}=\partial_{6} B_{i j}, \quad H_{i j k}=3 \partial_{[i} B_{j k]}$. Separating the $5 \mathrm{~d}$ transverse part as $B_{i j}=B_{i j}^{\perp}+\partial_{i} b_{j}-\partial_{j} b_{i}$ and integrating over $b_{i}$ one finds that the resulting determinant cancels against the ghost and Jacobian factors and we end up with

$$
Z=\frac{1}{\left[\operatorname{det} \Delta_{\perp}\right]^{1 / 2}}
$$

where $\Delta_{\perp}$ is the 6 d Laplacian defined on $B_{i j}^{\perp}$. Thus (A.4) describes $\frac{1}{2} \times 4 \times 5-(5-1)=6$ degrees of freedom (for similar discussion in the $4 \mathrm{~d}$ vector case see eqs. (2.14), (2.15) in [34]).

Analogous considerations in the self-dual case described by the classical actions (2.19) or (2.23) lead to the partition function (2.22) or the "square root" of (A.4) (cf. (2.25)).

\section{B Useful integrals}

We use the following standard integrals:

$$
\begin{aligned}
\int \frac{d^{d} q}{(2 \pi)^{d}} \frac{1}{\left(q^{2}+X\right)^{m}} & =\frac{1}{(4 \pi)^{d / 2}} \frac{\Gamma(m-d / 2)}{\Gamma(m)} \frac{1}{X^{m-d / 2}} \\
\int \frac{d^{d} q}{(2 \pi)^{d}} \frac{q^{i} q^{j}}{\left(q^{2}+X\right)^{n}} & =\frac{1}{d} \int \frac{d^{d} q}{(2 \pi)^{d}} \frac{q^{2} \delta^{i j}}{\left(q^{2}+X\right)^{n}}, \\
\int \frac{d^{d} q}{(2 \pi)^{d}} \frac{q^{i} q^{j} q^{k} q^{l}}{\left(q^{2}+X\right)^{n}} & =\frac{1}{d(d+2)} \int \frac{d^{d} q}{(2 \pi)^{d}} \frac{\left(q^{2}\right)^{2}\left(\delta^{i j} \delta^{k l}+\delta^{i k} \delta^{l j}+\delta^{i l} \delta^{j k}\right)}{\left(q^{2}+X\right)^{n}}
\end{aligned}
$$

and the identity

$$
\int \frac{d^{d} q}{(2 \pi)^{d}} \frac{q^{2 n}}{\left(q^{2}+X\right)^{m}}=-\frac{d+2(n-1)}{d+2(n-1)-2(m-1)} X \int \frac{d^{d} q}{(2 \pi)^{d}} \frac{q^{2 n-2}}{\left(q^{2}+X\right)^{m}} .
$$

The integral used for evaluation of one-dimensional integrals over $p_{6}$ is

$$
\int_{-\infty}^{+\infty} d \mu \frac{\mu^{2 n}}{\left(1+\mu^{2}\right)^{m-d / 2}}=\frac{\Gamma(m-n-(d+1) / 2) \Gamma(n+1 / 2)}{\Gamma(m-d / 2)}, \quad m, n \in \mathbf{Z} .
$$

\footnotetext{
${ }^{24}$ Note that $\operatorname{det} \Delta_{1}=\Delta_{1 \perp} \operatorname{det} \Delta_{0}, \quad \operatorname{det} \Delta_{2}=\operatorname{det} \Delta_{2 \perp} \operatorname{det} \Delta_{1 \perp}$.
} 


\section{C $\quad A^{6}$ term in effective action of self-dual B-field}

To obtain the $\beta_{2}$ and $\beta_{3}$ coefficients in the divergent part of the effective action (3.2) one may either compute the derivative-dependent $A^{2}$ and $A^{3}$ terms as was done in section 4 , or consider a constant non-abelian $A_{i}^{a}$-field and find the coefficients of the independent $A^{6}$ terms in $\Gamma$. For constant potential one has $F_{i j}^{a}=f^{a b c} A_{i}^{b} A_{j}^{c}$ and thus

$$
\begin{aligned}
D_{j} F_{i j}^{a} D_{k} F_{i k}^{a} & =f^{a b c} f^{c d e} f^{a f h} f^{h g w} A_{j}^{b} A_{i}^{d} A_{j}^{e} A_{k}^{f} A_{i}^{g} A_{k}^{w}, \\
f^{a d g} F_{i j}^{a} F_{j k}^{d} F_{k i}^{g} & =f^{a d g} f^{a b c} f^{d e f} f^{g h w} A_{i}^{b} A_{j}^{c} A_{j}^{e} A_{k}^{f} A_{k}^{h} A_{i}^{w} .
\end{aligned}
$$

It is sufficient to consider the $\mathrm{SU}(2)$ case where $f_{a b c}=\epsilon_{a b c}(a=1,2,3)$. The effective action corresponding to the self-dual model (4.1), (4.2), (4.3) in a constant non-abelian $\mathrm{SU}(2)$ background potential $A_{i}^{a}$ may be written as

$$
\Gamma_{+}=\frac{1}{2} \int d^{6} x \int \frac{d^{6} p}{(2 \pi)^{6}} \operatorname{tr} \ln \left[1+\left(\Delta^{(0)}(p)\right)^{-1} \Delta^{(1)}(p)\right],
$$

where the propagator $\left(\Delta^{(0)}\right)^{-1}$ and the vertex $\Delta^{(1)}$ (linear in $A$ ) are given by (4.5), (4.6) in momentum representation. As a result, the $A^{6}$ structures coming out of the terms $\sim\left(P_{i j}^{m n} \epsilon_{m n r k l} A_{r}^{a} p_{6}\right)^{6}$ are given by

$$
\begin{aligned}
\Gamma_{6}=\int d^{6} x \int & \frac{d p_{6}}{2 \pi} \frac{d^{d} p}{(2 \pi)^{d}} \frac{1}{\left(p^{2}+p_{6}^{2}\right)^{6} p_{6}^{2}}\left[\frac{1}{35}\left(I_{1}+3 I_{2}-4 I_{3}\right) p^{8}\right. \\
& -\frac{1}{3}\left(\frac{221}{210} I_{1}-\frac{47}{14} I_{2}+\frac{7}{3} I_{3}\right) p^{8}+\left(\frac{3}{10} I_{1}+\frac{9}{14} I_{2}-\frac{36}{35} I_{3}\right) p^{4} p_{6}^{4} \\
& \left.+\left(\frac{7}{10} I_{1}-\frac{3}{2} I_{2}+\frac{1}{5} I_{3}\right) g p^{2} p_{6}^{6}+\left(\frac{5}{6} I_{1}-\frac{1}{2} I_{2}+\frac{2}{3} I_{3}\right) p_{6}^{8}\right],
\end{aligned}
$$

where $p^{2}=p_{i} p_{i}$ and the 6 -volume factorizes. We introduced the following notations for the $A^{6}$ contractions

$$
I_{1}=Q^{a c} Q^{a b} Q^{b c}, \quad I_{2}=Q^{a a} Q^{b c} Q^{b c}, \quad I_{3}=Q^{a a} Q^{b b} Q^{c c}, \quad Q^{a b} \equiv A_{i}^{a} A_{i}^{b},
$$

in terms of which the two invariants in (C.1) have the forms

$$
F^{3} \equiv f^{a d g} F_{i j}^{a} F_{j k}^{d} F_{k i}^{g}=2 I_{1}-3 I_{2}+I_{3}, \quad(D F)^{2} \equiv D_{j} F_{i j}^{a} D_{k} F_{i k}^{a}=I_{1}-2 I_{2}+I_{3} .
$$

Using (B.4) we can rewrite the integrals in (C.3) as (setting $d=5$ in the coefficients)

$$
\int d^{d} p \frac{1}{\left(p^{2}+p_{6}^{2}\right)^{6} p_{6}^{2}}\left\{p^{8}, p^{8}, p^{4} p_{6}^{4}, p^{2} p_{6}^{6}\right\}=\left\{-231,21, \frac{7}{3}, 1\right\} \int d^{d} p \frac{p_{6}^{6}}{\left(p^{2}+p_{6}^{2}\right)^{6}} .
$$

Then the effective action (C.3) takes the form consistent with gauge invariance (cf. (C.5))

$$
\begin{aligned}
\Gamma_{6} & =-\frac{16}{15} c \int d^{6} x\left(11 I_{1}-3 I_{2}-8 I_{3}\right)=\frac{16}{15} c \int d^{6} x\left[27(D F)^{2}-19 F^{3}\right], \\
c & \equiv \int \frac{d p_{6}}{2 \pi} \frac{d^{d} p}{(2 \pi)^{d}} \frac{p_{6}^{6}}{\left(p^{2}+p_{6}^{2}\right)^{6}}=-\frac{1}{2^{11} \pi^{3}(d-5)}+\ldots .
\end{aligned}
$$


Here to isolate the UV logarithmic divergence we integrated over $p_{6}$, used (B.1) and took $d \rightarrow 5$ ignoring IR singularity (which is related to the expansion in powers of constant $A$ )

$$
\int_{-\infty}^{\infty} \frac{d p_{6}}{2 \pi} \frac{p_{6}^{6}}{\left(p^{2}+p_{6}^{2}\right)^{6}}=\frac{3}{512 p^{5}}, \quad \int \frac{d^{d} p}{(2 \pi)^{d}} \frac{1}{p^{5}} \rightarrow \frac{1}{(4 \pi)^{d / 2}} \frac{\Gamma\left(\frac{5-d}{2}\right)}{\Gamma\left(\frac{5}{2}\right)}=-\frac{1}{12 \pi^{3}(d-5)}+\ldots
$$

Thus finally

$$
\Gamma_{6 \infty}=-\frac{1}{2^{7} \pi^{3}(d-5)} \int d^{6} x\left[\frac{9}{5}(D F)^{2}-\frac{19}{15} F^{3}\right]
$$

Using that $\frac{1}{d-5} \rightarrow-\log \Lambda$ and that $C_{2}=2$ in the $\mathrm{SU}(2)$ case this can be written also as

$$
\Gamma_{6 \infty}=\frac{1}{2^{8} \pi^{3}} C_{2} \log \Lambda \int d^{6} x\left[\frac{9}{5}(D F)^{2}-\frac{19}{15} F^{3}\right]
$$

which is consistent with (3.1), (3.2), (3.3), (3.4) for the same values of the coefficients

$$
\beta_{2}=-27, \quad \beta_{3}=-57,
$$

as found in section 4 .

\section{Non-abelian chiral $4 \mathrm{~d}$ vector model}

Here we consider a $4 \mathrm{~d}$ analog of the $6 \mathrm{~d}$ self-dual $B$-field model (2.27) where the tensor field $B_{i j}$ is replaced by a vector $B_{i}$ (with $i=1,2,3$ ) which is coupled to a gauge field living in a 3 d subspace (or a "defect"):

$$
S_{4}=\int d x_{4} d^{3} x\left[\left(\partial_{4} B_{i}^{a}\right)^{2}+i m \epsilon_{i j k} B_{i}^{a} D_{k} B_{j}^{a}\right], \quad D_{k} B_{i}^{a}=\partial_{k} B_{i}^{a}+f^{a b c} A_{k}^{b} B_{i}^{c} .
$$

We assume that $B_{i}$ is in adjoint representation and depends on all 4 coordinates while $A_{i}^{a}$ depends only on 3 coordinates $x_{i}$. Here we can not have $\partial_{4}$ in the $\epsilon_{i j k}$ term as otherwise this term vanishes, so we need to introduce a mass parameter $m$ to balance the dimensions. The action is invariant under the local symmetry (with $U=U\left(x_{i}\right)$, cf. (2.17))

$$
B_{i}^{\prime}=U B_{i} U^{-1}, \quad A_{i}^{\prime}=U\left(A_{i}+\partial_{i}\right) U^{-1}
$$

Integrating out the $B_{i}$-field one should then get a gauge-invariant effective action $\Gamma(A)$ depending on the $3 \mathrm{~d}$ field $A_{i}$. Since the classical action is not parity-invariant, $\Gamma$ may contain a non-local P-odd part.

The analogs of the propagator (4.5) and the vertex (4.6) linear in $A$ here are $\left(p^{2}=p_{i} p_{i}\right)$

$$
P_{i j}^{a b}=\delta^{a b} P_{i j}\left(p_{s}, p_{4}\right)=\frac{\delta^{a b}}{m^{2} p^{2}+p_{4}^{4}}\left(p_{4}^{2} \delta_{i j}-m \epsilon_{i j k} p_{k}+\frac{m^{2}}{p_{4}^{2}} p_{i} p_{j}\right), \quad V_{i j k}^{a b c}=-m \epsilon_{i j k} f^{a b c}
$$

To compute the effective action we use dimensional regularization in a $3 \mathrm{~d}$ variant of the $4 \mathrm{~d}$ helicity scheme, in which all the numerator algebra is carried out in 3 dimensions and 
then the remaining scalar momentum integrals are done in $d=3-2 \epsilon$ dimensions (with $p_{4}$ integral treated as 1-dimensional one).

The one-loop two-point function of the external $A_{i}^{a}$-field appearing in the $A^{2}$ term of the effective action may be written as ( $k_{s}$ is an external 3 -momentum)

$$
\begin{aligned}
& i \Pi_{i j}^{a b}\left(k_{s}\right)=\int \frac{d p_{4}}{2 \pi} \frac{d^{d} p}{(2 \pi)^{d}} V_{m n i}^{c d a} P_{n r}^{d e}\left(p_{s}, p_{4}\right) V_{r q j}^{e f b} P_{q m}^{f c}\left(p_{s}+k_{s}, p_{4}\right)=i \delta^{a b} \Pi_{i j}\left(k_{s}\right), \\
& i \Pi_{i j}\left(k_{s}\right)=-m^{2} C_{2} \epsilon_{i m n} \epsilon_{j p r} \int \frac{d p_{4}}{2 \pi} \frac{d^{d} p}{(2 \pi)^{d}} P_{n r}\left(p_{i}, p_{4}\right) P_{q m}\left(p_{i}+k_{i}, p_{4}\right) .
\end{aligned}
$$

Using the identity (B.4) with $n=1$ (which is equivalent to $\int \frac{d^{d} q}{(2 \pi)^{d}} \frac{\partial}{\partial q^{\mu}} \frac{q_{\mu}}{q^{2}+X}=0$ ) and introducing the Feynman parameter $y$ leads to

$$
\begin{aligned}
i \Pi_{i j}\left(k_{s}\right) & =-m^{2} C_{2} \int_{0}^{1} d y \int \frac{d p_{4}}{2 \pi} \frac{d^{d} p}{(2 \pi)^{d}} \frac{N_{i j}\left(k_{s}, p_{4}, y\right)}{p_{4}^{4}\left[m^{2} p^{2}+p_{4}^{4}+m^{2} y(1-y) k^{2}\right]^{2}}, \\
N_{i j} & =m^{2} y(1-y)\left(4 p_{4}^{4}+m^{2} k^{2}\right)\left(k^{2} \delta_{i j}-k_{i} k_{j}\right)-2 m \frac{d-1}{d-2} p_{4}^{2}\left[p_{4}^{4}+m^{2} y(1-y) k^{2}\right] \epsilon_{i j s} k_{s} .
\end{aligned}
$$

Performing the $p_{i}$ integral using (B.1) gives

$$
i \Pi_{i j}=-m^{2-d} C_{2} \frac{\Gamma(2-d / 2)}{(4 \pi)^{d / 2}} \int_{0}^{1} d y \int \frac{d p_{4}}{2 \pi} \frac{N_{i j}\left(k_{s}, p_{4}, y\right)}{p_{4}^{4}\left[p_{4}^{4}+m^{2} y(1-y) k^{2}\right]^{2-d / 2}} .
$$

To integrate over $p_{4}$ we first change the variable to $\mu$ as $p_{4}=\mu\left[m^{2} y(1-y) k^{2}\right]^{1 / 4}$ and then use (B.5). Integrating over $y$ we finally obtain

$$
i \Pi_{i j}=-\frac{1}{30 \pi}\left(\frac{m^{2}}{k^{2}}\right)^{1 / 4}\left(k^{2} \delta_{i j}-k_{i} k_{j}\right)-\frac{1}{6 \pi}\left(m^{2} k^{2}\right)^{1 / 4} \epsilon_{i j r} k_{r} .
$$

In contrast to the $6 \mathrm{~d}$ case in (4.7)-(4.11), here the $A^{2}$ term in the effective action is UV finite and contains two $3 \mathrm{~d}$ gauge-invariant non-local structures: P-even one $\int F_{i j}\left(\frac{m^{2}}{\partial^{2}}\right)^{1 / 4} F_{i j}$ and P-odd one $\int \epsilon_{k i j} A_{k}\left(m^{2} \partial^{2}\right)^{1 / 4} F_{i j}$.

Open Access. This article is distributed under the terms of the Creative Commons Attribution License (CC-BY 4.0), which permits any use, distribution and reproduction in any medium, provided the original author(s) and source are credited.

\section{References}

[1] C. Sämann and L. Schmidt, Towards an M5-brane model I: a 6d superconformal field theory, J. Math. Phys. 59 (2018) 043502 [arXiv:1712.06623] [INSPIRE].

[2] H. Samtleben, E. Sezgin and R. Wimmer, $(1,0)$ superconformal models in six dimensions, JHEP 12 (2011) 062 [arXiv: 1108.4060] [INSPIRE].

[3] H. Samtleben, E. Sezgin and R. Wimmer, Six-dimensional superconformal couplings of non-abelian tensor and hypermultiplets, JHEP 03 (2013) 068 [arXiv:1212.5199] [INSPIRE]. 
[4] H. Samtleben, E. Sezgin, R. Wimmer and L. Wulff, New superconformal models in six dimensions: gauge group and representation structure, POS (CORFU2011) 071 [arXiv: 1204.0542] [INSPIRE].

[5] N. Lambert and C. Papageorgakis, Nonabelian $(2,0)$ tensor multiplets and 3-algebras, JHEP 08 (2010) 083 [arXiv: 1007.2982] [INSPIRE].

[6] C.-S. Chu and S.-L. Ko, Non-abelian action for multiple five-branes with self-dual tensors, JHEP 05 (2012) 028 [arXiv: 1203.4224] [INSPIRE].

[7] F. Bonetti, T.W. Grimm and S. Hohenegger, Non-abelian tensor towers and $(2,0)$ superconformal theories, JHEP 05 (2013) 129 [arXiv: 1209.3017] [INSPIRE].

[8] P.-M. Ho, K.-W. Huang and Y. Matsuo, A non-abelian self-dual gauge theory in $5+1$ dimensions, JHEP 07 (2011) 021 [arXiv:1104.4040] [INSPIRE].

[9] P.-M. Ho and Y. Matsuo, Note on non-Abelian two-form gauge fields, JHEP 09 (2012) 075 [arXiv: 1206.5643] [INSPIRE].

[10] P.-M. Ho and Y. Matsuo, Aspects of effective theory for multiple M5-branes compactified on circle, JHEP 12 (2014) 154 [arXiv:1409.4060] [INSPIRE].

[11] K.W. Huang, Non-Abelian chiral 2-form and M5-branes, arXiv:1206.3983.

[12] E.S. Fradkin and A.A. Tseytlin, Quantum properties of higher dimensional and dimensionally reduced supersymmetric theories, Nucl. Phys. B 227 (1983) 252 [INSPIRE].

[13] P.H. Frampton and T.W. Kephart, Explicit Evaluation of Anomalies in Higher Dimensions, Phys. Rev. Lett. 50 (1983) 1343 [Erratum ibid. 51 (1983) 232] [INSPIRE].

[14] L. Álvarez-Gaumé and E. Witten, Gravitational anomalies, Nucl. Phys. B 234 (1984) 269 [INSPIRE].

[15] F. Bastianelli and P. van Nieuwenhuizen, Gravitational anomalies from the action for selfdual antisymmetric tensor fields in $(4 k+2)$-dimensions, Phys. Rev. Lett. 63 (1989) 728 [INSPIRE].

[16] M. Beccaria and A.A. Tseytlin, Partition function of free conformal fields in 3-plet representation, JHEP 05 (2017) 053 [arXiv:1703.04460] [INSPIRE].

[17] M. Henneaux and C. Teitelboim, Dynamics of chiral (selfdual) P forms, Phys. Lett. B 206 (1988) 650 [INSPIRE].

[18] J.H. Schwarz and A. Sen, Duality symmetric actions, Nucl. Phys. B 411 (1994) 35 [hep-th/9304154] [INSPIRE].

[19] M. Perry and J.H. Schwarz, Interacting chiral gauge fields in six-dimensions and Born-Infeld theory, Nucl. Phys. B 489 (1997) 47 [hep-th/9611065] [INSPIRE].

[20] P. Pasti, D.P. Sorokin and M. Tonin, On Lorentz invariant actions for chiral $p$ forms, Phys. Rev. D 55 (1997) 6292 [hep-th/9611100] [INSPIRE].

[21] D. Belov and G.W. Moore, Holographic action for the self-dual field, hep-th/0605038 [INSPIRE].

[22] P.B. Gilkey, The spectral geometry of a Riemannian manifold, J. Diff. Geom. 10 (1975) 601.

[23] F. Bastianelli, S. Frolov and A.A. Tseytlin, Conformal anomaly of $(2,0)$ tensor multiplet in six-dimensions and AdS/CFT correspondence, JHEP 02 (2000) 013 [hep-th/0001041] [INSPIRE]. 
[24] H. Osborn and A. Stergiou, Structures on the conformal manifold in six dimensional theories, JHEP 04 (2015) 157 [arXiv: 1501.01308] [INSPIRE].

[25] E.A. Ivanov, A.V. Smilga and B.M. Zupnik, Renormalizable supersymmetric gauge theory in six dimensions, Nucl. Phys. B 726 (2005) 131 [hep-th/0505082] [INSPIRE].

[26] A.V. Smilga, Chiral anomalies in higher-derivative supersymmetric $6 D$ theories, Phys. Lett. B 647 (2007) 298 [hep-th/0606139] [INSPIRE].

[27] I.L. Buchbinder et al., One-loop divergences in $6 D, \mathcal{N}=(1,0)$ SYM theory, JHEP 01 (2017) 128 [arXiv: 1612.03190] [INSPIRE].

[28] I.L. Buchbinder, E.A. Ivanov, B.S. Merzlikin and K.V. Stepanyantz, Supergraph analysis of the one-loop divergences in $6 D, \mathcal{N}=(1,0)$ and $\mathcal{N}=(1,1)$ gauge theories, Nucl. Phys. B 921 (2017) 127 [arXiv: 1704. 02530] [INSPIRE].

[29] E. Ivanov, Classical and quantum superfield invariants in $\mathcal{N}=(1,1), 6 D$ SYM theory, $J$. Phys. Conf. Ser. 965 (2018) 012021.

[30] J. Bhattacharya and A.E. Lipstein, $6 d$ dual conformal symmetry and minimal volumes in AdS, JHEP 12 (2016) 105 [arXiv: 1611.02179] [INSPIRE].

[31] R. Manvelyan and A.C. Petkou, The trace anomaly of the $(2,0)$ tensor multiplet in background gauge fields, JHEP 06 (2000) 003 [hep-th/0005256] [INSPIRE].

[32] A.S. Schwarz, The partition function of a degenerate functional, Commun. Math. Phys. 67 (1979) 1 [INSPIRE].

[33] A.S. Schwarz and Yu.S. Tyupkin, Quantization of antisymmetric tensors and Ray-Singer torsion, Nucl. Phys. B 242 (1984) 436 [INSPIRE].

[34] M. Beccaria, X. Bekaert and A.A. Tseytlin, Partition function of free conformal higher spin theory, JHEP 08 (2014) 113 [arXiv: 1406.3542] [INSPIRE]. 